Check for updates

Cite this: RSC Adv., 2018, 8, 13592

Received 17th January 2018

Accepted 3rd April 2018

DOI: $10.1039 / c 8 r a 00506 k$

rsc.li/rsc-advances

\title{
Well-defined benzoxazine/triphenylamine-based hyperbranched polymers with controlled degree of branchingt
}

\author{
Ruey-Chorng Lin and Shiao-Wei Kuo (D)*
}

\begin{abstract}
Well-defined thermally polymerizable hyperbranched polymers (TPA-BZs) containing various numbers of triphenylamine (TPA) and benzoxazine (BZ) units have been prepared using a "click-like" reaction concept, through one-pot Mannich condensations of 4-(bis(4-aminophenyl)amino)phenol (TPA-2 $\mathrm{NH}_{2}-$ $\mathrm{OH}$, as the $\mathrm{AB}_{2}$ branching groups), aniline (as the focal groups), $\mathrm{CH}_{2} \mathrm{O}$, and phenol in 1,4-dioxane, with a unique feeding approach. Two design strategies for the chemical construction were applied: (i) simple hyperbranched TPA-BZs, such as those containing one or three TPA units, developed from the focal or the terminal group direction to form the resultant monomers; (ii) three dendritic TPA-BZs containing four TPA units possessing different degrees of branching (DBs) for the conformation study. The exothermic temperature for the dendritic TPA-BZs decreased upon increasing the DB. The bathochromic shifts of the dendritic TPA-BZs increased upon increasing the number of TPA units, in UV-Vis absorption and PL emission spectra, presumably because of an increase in the effective conjugation length. In addition, the polymerized dendritic TPA-BZ DG1 possessed thermal properties superior to those of the hyperbranched TPA-BZ polybenzoxazines, possibly because the segmental mobility in the polymer network was restricted by the dendrimer core group and because of its symmetrical construction. The hyperbranched TPA-BZ possessed unique photophysical properties, suggesting potential applications in optoelectronic devices.
\end{abstract}

\section{Introduction}

Hyperbranched polymers are asymmetrical counterparts to symmetrical dendrimers, but possess many of the unique features of dendrimers, including low viscosity, good solubility, terminal group functionality, and high branching density. ${ }^{\mathbf{1 , 2}}$ In contrast to perfect dendrimers with uniform chemical constructions, the merits of imperfect hyperbranched polymers are often underestimated because they have some unattractive features-for example, uncontrolled molecular weight, broad distribution, and diversity in their chemical construction; in some cases they considered only the results as side reactions in polymerizations or as network formation precursors of dendrimers. $^{3}$ The applications of dendrimers are, however, restricted by their high cost and difficult production on large scales, due to tedious multistep reactions and complicated syntheses., ${ }^{\mathbf{4} 5}$ Here, imperfect hyperbranched polymers can overcome the disadvantages of dendrimers because they are more conveniently produced (e.g., through one-pot syntheses); ${ }^{6}$ accordingly, they are more popular than dendrimers in some

Department of Materials and Optoelectronic Science, National Sun Yat-Sen University, Kaohsiung 80424, Taiwan.E-mail: kuosw@faculty.nsysu.edu.tw

$\uparrow$ Electronic supplementary information (ESI) available. See DOI: $10.1039 / \mathrm{c} 8 \mathrm{ra00506k}$ applications (e.g., sensor devices, special additive carriers, biology, blending, painting, coating). ${ }^{3-7}$

In general, two main approaches are used for the preparation of hyperbranched polymers: the single-monomer methodology, which involves polymerization of an $\mathrm{AB}_{n}$ monomer or an $\mathrm{AB}_{n}$ analogue, and the double-monomer methodology, which involves polymerization of two types of monomers or a monomer pair (e.g., an $\mathrm{A}_{2}+\mathrm{B}_{y}$ monomer combination), employing several common reactions (e.g., ring-opening multi-branching polymerization; self-condensing vinyl polymerization; selfcondensing ring-opening polymerization)., ${ }^{2,8}$ Over the past two decades, much research effort has been dedicated to controlled syntheses for preparing hyperbranched polymers with precise degrees of branching (DBs), ${ }^{9-16}$ chemical conformations from linear to hyperbranched, ${ }^{17-19}$ DBs from 0 to $100 \%,{ }^{20}$ and controlled molecular weights and distributions, ${ }^{21-28}$ in an effort to overcome the negative stigma associated with their perceived uncontrolled features. Hence, the development of well-defined and well-controlled hyperbranched polymers is an important step in their increased utility in academic studies and industrial applications., ${ }^{3,29}$

Benzoxazine (BZ) monomers possess the ability to undergo thermal ring-opening polymerization, without the need for any catalysts and without the formation of byproducts, forming polymers with several unique features (e.g., high chemical 
resistance, low surface free energy, high flame retardancy) that have drawn the attention of both academia and industry. ${ }^{30-37}$ Triphenylamine (TPA) possesses a unique two-photon absorption ability and has been studied and applied in many fields, including optoelectronic devices and electron transition devices. ${ }^{\mathbf{3 8 - 4 0}}$ Hence, we have been interested in combining the properties of BZ and TPA units in polymeric systems. In a previous study, we synthesized a series of BZ/TPA-based dendrimers [e.g., a generation-three dendrimer (TPA-BZ DG3) possessing 22 TPA and $45 \mathrm{BZ}$ units] through facile one-pot Mannich condensations. ${ }^{41}$ Interestingly, the small bathochromic shifts were observed in UV-Vis absorption and PL emission spectra upon increasing the number of TPA units, the result of an effective increase in the conjugation length. ${ }^{42,43}$ The symmetrical TPA-BZ dendrimers were constructed using TPA$\mathrm{BZ}$ dendrons (asymmetrical hyperbranched monomers); we were interested in comparing the properties of the dendrons of symmetrical dendrimers and their corresponding hyperbranched monomers. The asymmetrical BZ monomers possess some properties that are very different from those of their symmetrical counterparts. Gu et al. reported that an asymmetrical monomer containing two BZ units exhibited characteristic signal splits in its ${ }^{1} \mathrm{H}$ nuclear magnetic resonance (NMR; oxazine rings) and Fourier transform infrared (FTIR; vibrations of trisubstituted benzene ring at $930 \mathrm{~cm}^{-1}$ ) spectra. ${ }^{44}$ Endo et al. also found that linear asymmetrical monomers, solely containing various numbers of BZ units, exhibited diversity in their characteristic signal splits (e.g., oxazine rings) in their ${ }^{1} \mathrm{H}$ and ${ }^{13} \mathrm{C}$ NMR spectra, depending on their chemical constructions. ${ }^{45,46}$ Hence, ${ }^{1} \mathrm{H}$ and ${ }^{13} \mathrm{C}$ NMR spectra can provide information about the chemical constructions of linear asymmetrical BZ monomers, due to the interactions of their adjacent groups, especially in the characteristic signal splits of the oxazine rings. We expected that the ${ }^{1} \mathrm{H}$ and ${ }^{13} \mathrm{C}$ NMR spectra of branching asymmetrical TPA-BZs would be affected more strongly than those of linear asymmetrical monomers because of the shorter distance between the adjacent groups; accordingly, it might be possible to monitor the complicated branching constructions of TPA-BZs in a very simple and straightforward approach. In addition, we have also investigated the photophysical properties of these asymmetrical hyperbranched TPA-BZ monomers.

In this study we synthesized a series of well-defined thermally polymerizable hyperbranched polymers (TPA-BZs), containing various numbers of TPA and BZ units, through facile one-pot Mannich condensations ${ }^{41}$ of TPA- $2 \mathrm{NH}_{2}-\mathrm{OH}$ (as the $\mathrm{AB}_{2}$ branching groups), aniline (as the focal groups), $\mathrm{CH}_{2} \mathrm{O}$, and phenol in 1,4-dioxane, using a unique feeding approach (Scheme 1 and $\mathrm{S} 1 \dagger$ display this "click-like" reaction concept). To understand the different chemical construction and the conformation of these hyperbranched TPA-BZs, we used two design strategies including (i) simple hyperbranched TPA-BZs, such as those containing one or three TPA units (i.e., TPA-BZ monomer and TPA-BZ trimer, respectively), developed from the focal or terminal group direction, as resultant monomers (Scheme 2); (ii) three dendritic TPA-BZs containing four TPA units (i.e., linear TPA-BZ tetramer, TPA-BZ tetramer, TPA-BZ
DG1) possessing different DBs (0-100\%) for our conformation study. ${ }^{47,48}$ This work is the first study to incorporate BZ units into well-defined hyperbranched TPA-based polymers with various conformations (DBs from 0 to $100 \%$ ) through such a convenient preparation strategy.

\section{Experimental section}

\section{Materials}

1-Fluoro-4-nitrobenzene (99\%), hydrazine monohydrate (99\%), trifluoroacetic anhydride (99\%), $p$-anisidine (99\%), cesium fluoride (99\%), 10\% Pd/C, charcoal, dimethyl sulfoxide (DMSO), dichloromethane, magnesium sulfate $\left(\mathrm{MgSO}_{4}\right)$, and tetrahydrofuran (THF) were purchased from Alfa-Aesar. Aniline (99.5\%), sodium borohydride (98\%), boron tribromide (99.9\%), sodium bicarbonate $\left(\mathrm{NaHCO}_{3}\right)$, paraformaldehyde, ethyl acetate, methanol $(\mathrm{MeOH})$, ethanol (EtOH), n-hexane, 1,4dioxane, and phenol (99\%) were purchased from SigmaAldrich. 4-(Bis(4-aminophenyl)amino)phenol (TPA-2 $\left.\mathrm{NH}_{2}-\mathrm{OH}\right)$ and TPA-BZ DG1 were prepared according to the literature. ${ }^{41}$

$N, N$-Bis(4-(2H-benzo[e][1,3]oxazin-3(4H)-yl)phenyl)-3-phenyl-3,4dihydro-2H-benzo $[e][1,3]$ oxazin-6-amine (TPA-BZ monomer). A solution of $\mathrm{CH}_{2} \mathrm{O}(0.103 \mathrm{~g}, 3.43 \mathrm{mmol})$ and aniline $(0.160 \mathrm{~g}, 1.72$ $\mathrm{mmol})$ in 1,4-dioxane $(30 \mathrm{~mL})$ was stirred under reflux at $90{ }^{\circ} \mathrm{C}$ for $2 \mathrm{~h}$ under $\mathrm{N}_{2}$ and then TPA-2 $\mathrm{NH}_{2}-\mathrm{OH}(0.500 \mathrm{~g}, 1.72 \mathrm{mmol})$ was added. After $24 \mathrm{~h}$ under reflux, $\mathrm{CH}_{2} \mathrm{O}(0.206 \mathrm{~g}, 6.87 \mathrm{mmol})$ was added. After $2 \mathrm{~h}$ under reflux, phenol $(0.323 \mathrm{~g}, 3.43 \mathrm{mmol})$ was added. After $24 \mathrm{~h}$ under reflux, the solution was cooled to room temperature and filtered. The filtrate was concentrated under reduced pressure and the residue taken up into $\mathrm{CH}_{2} \mathrm{Cl}_{2}(3 \times 80 \mathrm{~mL})$. The organic solution was washed several times with saturated aqueous $\mathrm{NaHCO}_{3}$ and distilled water, dried $\left(\mathrm{MgSO}_{4}, 30 \mathrm{~min}\right)$, and concentrated. The residue was purified through column chromatography $\left(\mathrm{SiO}_{2} ; \mathrm{CH}_{2} \mathrm{Cl}_{2} / \mathrm{MeOH}, 1: 2\right)$ to yield a brown product (0.76 g, 69\%): FTIR (KBr, cm $\left.{ }^{-1}\right): 1499,1337,1227,941 .{ }^{1} \mathrm{H}$ NMR (500 MHz, DMSO- $d_{6}, \delta$, ppm): 6.42-7.53 (m, aromatic $\left.\mathrm{CH}\right), 5.36$ and 5.42 (s, $\left.2 \mathrm{H}, \mathrm{OCH}_{2} \mathrm{~N}\right), 4.58$ and $4.63\left(\mathrm{~s}, 2 \mathrm{H}, \mathrm{CCH}_{2} \mathrm{~N}\right) \cdot{ }^{13} \mathrm{C}$ NMR $(125 \mathrm{MHz}$, DMSO- $d_{6}, \delta$, ppm): 153.96 (peak a), 151.14 (peak b), 147.82 (peak c), 143.63 (peak d), 115.24-153.96 (aromatic), 78.66 and $79.21\left(\mathrm{OCH}_{2} \mathrm{~N}\right)$, 48.90 and $49.40\left(\mathrm{CCH}_{2} \mathrm{~N}\right)$. MALDI-TOF $\mathrm{MS}:[\mathrm{M}]^{+}(\mathrm{m} / \mathrm{z})$ for $\left(\mathrm{C}_{42} \mathrm{H}_{36} \mathrm{~N}_{4} \mathrm{O}_{3}\right)$ : 644.99: calc., 644.76 (Fig. S1 $\dagger$ ).

\section{TPA-BZ trimer}

A solution of $\mathrm{CH}_{2} \mathrm{O}(0.0310 \mathrm{~g}, 1.03 \mathrm{mmol})$ and aniline $(0.0480 \mathrm{~g}$, $0.515 \mathrm{mmol}$ ) in 1,4-dioxane (30 mL) was stirred under reflux at $90{ }^{\circ} \mathrm{C}$ for $2 \mathrm{~h}$ under $\mathrm{N}_{2}$ and then TPA- $2 \mathrm{NH}_{2}-\mathrm{OH}(0.150 \mathrm{~g}, 0.515$ mmol) was added. After $24 \mathrm{~h}$ under reflux, $\mathrm{CH}_{2} \mathrm{O}(0.0620 \mathrm{~g}, 2.06$ mmol) was added. After $2 \mathrm{~h}$ under reflux, TPA- $2 \mathrm{NH}_{2}-\mathrm{OH}$ (0.300 g, $1.03 \mathrm{mmol}$ ) was added. After $24 \mathrm{~h}$ under reflux, $\mathrm{CH}_{2} \mathrm{O}$ $(0.124 \mathrm{~g}, 4.12 \mathrm{mmol})$ was added. After $2 \mathrm{~h}$ under reflux, phenol $(0.194 \mathrm{~g}, 2.06 \mathrm{mmol})$ was added. After $24 \mathrm{~h}$ under reflux, the solution was cooled to room temperature and filtered. The filtrate was concentrated under reduced pressure and the residue taken up into $\mathrm{CH}_{2} \mathrm{Cl}_{2}(3 \times 80 \mathrm{~mL})$. The organic solution was washed several times with saturated aqueous $\mathrm{NaHCO}_{3}$ and distilled water, dried $\left(\mathrm{MgSO}_{4}, 30 \mathrm{~min}\right)$, and concentrated. The 
(A)

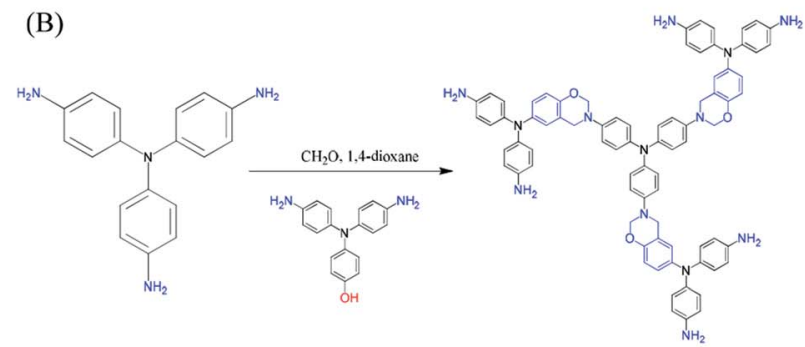

(a)

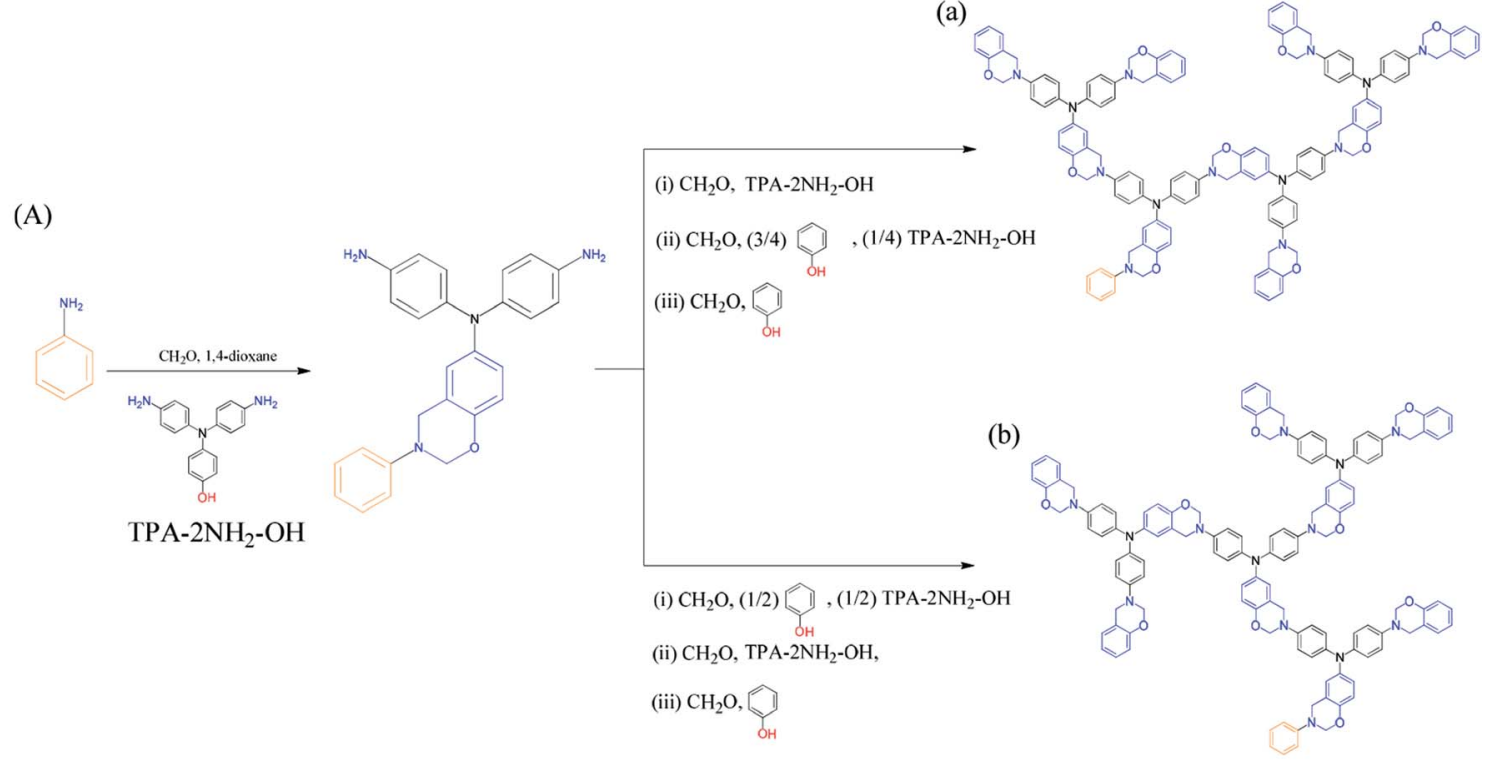

(i) $\mathrm{CH}_{2} \mathrm{O}, 0$ (c)

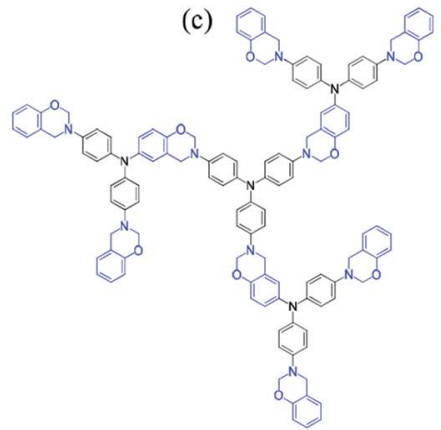

Scheme 1 Synthesis of (A) the hyperbranched TPA-BZs (a) linear-tetramer and (b) tetramer and (B) the TPA-BZ dendrimer (c) TPA-BZ DG1.

residue was purified through column chromatography $\left(\mathrm{SiO}_{2}\right.$; $\left.\mathrm{CH}_{2} \mathrm{Cl}_{2} / \mathrm{MeOH}, 1: 2\right)$ to yield a brown product $(0.55 \mathrm{~g}, 70 \%)$ : FTIR $\left(\mathrm{KBr}, \mathrm{cm}^{-1}\right)$ : 1499, 1337, 1227, 941. ${ }^{1} \mathrm{H}$ NMR $(500 \mathrm{MHz}$, DMSO- $\left.d_{6}, \delta, \mathrm{ppm}\right): 6.39-7.55$ (m, $\mathrm{CH}$ aromatic), 5.36 and 5.43 (s, $\left.2 \mathrm{H}, \mathrm{OCH}_{2} \mathrm{~N}\right), 4.58$ and $4.64\left(\mathrm{~s}, 2 \mathrm{H}, \mathrm{CCH}_{2} \mathrm{~N}\right) \cdot{ }^{13} \mathrm{C} \mathrm{NMR}(125 \mathrm{MHz}$, DMSO- $d_{6}, \delta$, ppm): 153.91 (peak a), 151.11 (peak b), 147.79 (peak c), 143.60 (peak d), 112.12-153.93 (aromatic), 78.63 and 79.18 $\left(\mathrm{OCH}_{2} \mathrm{~N}\right), 48.87$ and $49.36\left(\mathrm{CCH}_{2} \mathrm{~N}\right)$.

\section{Linear TPA-BZ tetramer}

A solution of $\mathrm{CH}_{2} \mathrm{O}(0.0260 \mathrm{~g}, 0.858 \mathrm{mmol})$ and aniline $(0.0400 \mathrm{~g}, 0.429 \mathrm{mmol})$ in 1,4-dioxane $(30 \mathrm{~mL})$ was stirred under reflux at $90{ }^{\circ} \mathrm{C}$ for $2 \mathrm{~h}$ under $\mathrm{N}_{2}$ and then TPA- $2 \mathrm{NH}_{2}-\mathrm{OH}$ $(0.125 \mathrm{~g}, 0.429 \mathrm{mmol})$ was added. After $24 \mathrm{~h}$ under reflux, $\mathrm{CH}_{2} \mathrm{O}$ $(0.0520 \mathrm{~g}, 1.72 \mathrm{mmol})$ was added. After $2 \mathrm{~h}$ under reflux, TPA$2 \mathrm{NH}_{2}-\mathrm{OH}(0.250 \mathrm{~g}, 0.858 \mathrm{mmol})$ was added. After $24 \mathrm{~h}$ under reflux, $\mathrm{CH}_{2} \mathrm{O}(0.103 \mathrm{~g}, 3.43 \mathrm{mmol})$ was added. After $2 \mathrm{~h}$ under reflux, phenol $(0.121 \mathrm{~g}, 1.29 \mathrm{mmol})$ was added. After $24 \mathrm{~h}$ under reflux, TPA-2 $\mathrm{NH}_{2}-\mathrm{OH}(0.125 \mathrm{~g}, 0.429 \mathrm{mmol})$ was added. After $24 \mathrm{~h}$ under reflux, $\mathrm{CH}_{2} \mathrm{O}(0.0520 \mathrm{~g}, 1.72 \mathrm{mmol})$ was added. After $2 \mathrm{~h}$ under reflux, phenol $(0.0810 \mathrm{~g}, 0.858 \mathrm{mmol})$ was added. After $24 \mathrm{~h}$ under reflux, the solution was cooled to room temperature and filtered. The filtrate was concentrated under reduced pressure and the residue taken up into $\mathrm{CH}_{2} \mathrm{Cl}_{2}(3 \times 80$ $\mathrm{mL})$. The organic solution was washed several times with saturated aqueous $\mathrm{NaHCO}_{3}$ and distilled water, dried $\left(\mathrm{MgSO}_{4}\right.$, $30 \mathrm{~min}$ ), and concentrated. The residue was purified through column chromatography $\left(\mathrm{SiO}_{2} ; \mathrm{CH}_{2} \mathrm{Cl}_{2} / \mathrm{MeOH}, 1 / 2\right)$ to yield a brown product $(0.54 \mathrm{~g}, 65 \%)$ : FTIR $\left(\mathrm{KBr}, \mathrm{cm}^{-1}\right): 1499,1339$, 1227, 941. ${ }^{1} \mathrm{H}$ NMR (500 MHz, DMSO- $\left.d_{6}, \delta, \mathrm{ppm}\right): 6.43-7.29$ (m, aromatic $\mathrm{CH}), 5.37$ and $5.43\left(\mathrm{~s}, 2 \mathrm{H}, \mathrm{OCH}_{2} \mathrm{~N}\right), 4.59$ and $4.64(\mathrm{~s}$, $\left.2 \mathrm{H}, \mathrm{CCH}_{2} \mathrm{~N}\right) \cdot{ }^{13} \mathrm{C}$ NMR $\left(125 \mathrm{MHz}, \mathrm{DMSO}-d_{6}, \delta, \mathrm{ppm}\right): 153.91$ (peak a), 151.11 (peak b), 147.79 (peak c), 143.60 (peak d), 116.19-153.93 (aromatic), 78.63 and $79.18\left(\mathrm{OCH}_{2} \mathrm{~N}\right), 48.87$ and $49.36\left(\mathrm{CCH}_{2} \mathrm{~N}\right)$.

\section{TPA-BZ tetramer}

A solution of $\mathrm{CH}_{2} \mathrm{O}(0.0260 \mathrm{~g}, 0.858 \mathrm{mmol})$ and aniline $(0.0400 \mathrm{~g}, 0.429 \mathrm{mmol})$ in 1,4-dioxane $(30 \mathrm{~mL})$ was stirred under reflux at $90{ }^{\circ} \mathrm{C}$ for $2 \mathrm{~h}$ under $\mathrm{N}_{2}$ and then TPA- $2 \mathrm{NH}_{2}-\mathrm{OH}$ $(0.125 \mathrm{~g}, 0.429 \mathrm{mmol})$ was added. After $24 \mathrm{~h}$ under reflux, $\mathrm{CH}_{2} \mathrm{O}$ $(0.0260 \mathrm{~g}, 0.858 \mathrm{mmol})$ was added. After $2 \mathrm{~h}$ under reflux, phenol $(0.0400 \mathrm{~g}, 0.429 \mathrm{mmol})$ was added. After $24 \mathrm{~h}$ under reflux, $\mathrm{CH}_{2} \mathrm{O}(0.0260 \mathrm{~g}, 0.858 \mathrm{mmol})$ was added. After $2 \mathrm{~h}$ under reflux, TPA- $2 \mathrm{NH}_{2}-\mathrm{OH}(0.125 \mathrm{~g}, 0.429 \mathrm{mmol})$ was added. After $24 \mathrm{~h}$ under reflux, $\mathrm{CH}_{2} \mathrm{O}(0.0510 \mathrm{~g}, 1.72 \mathrm{mmol})$ was added. After 

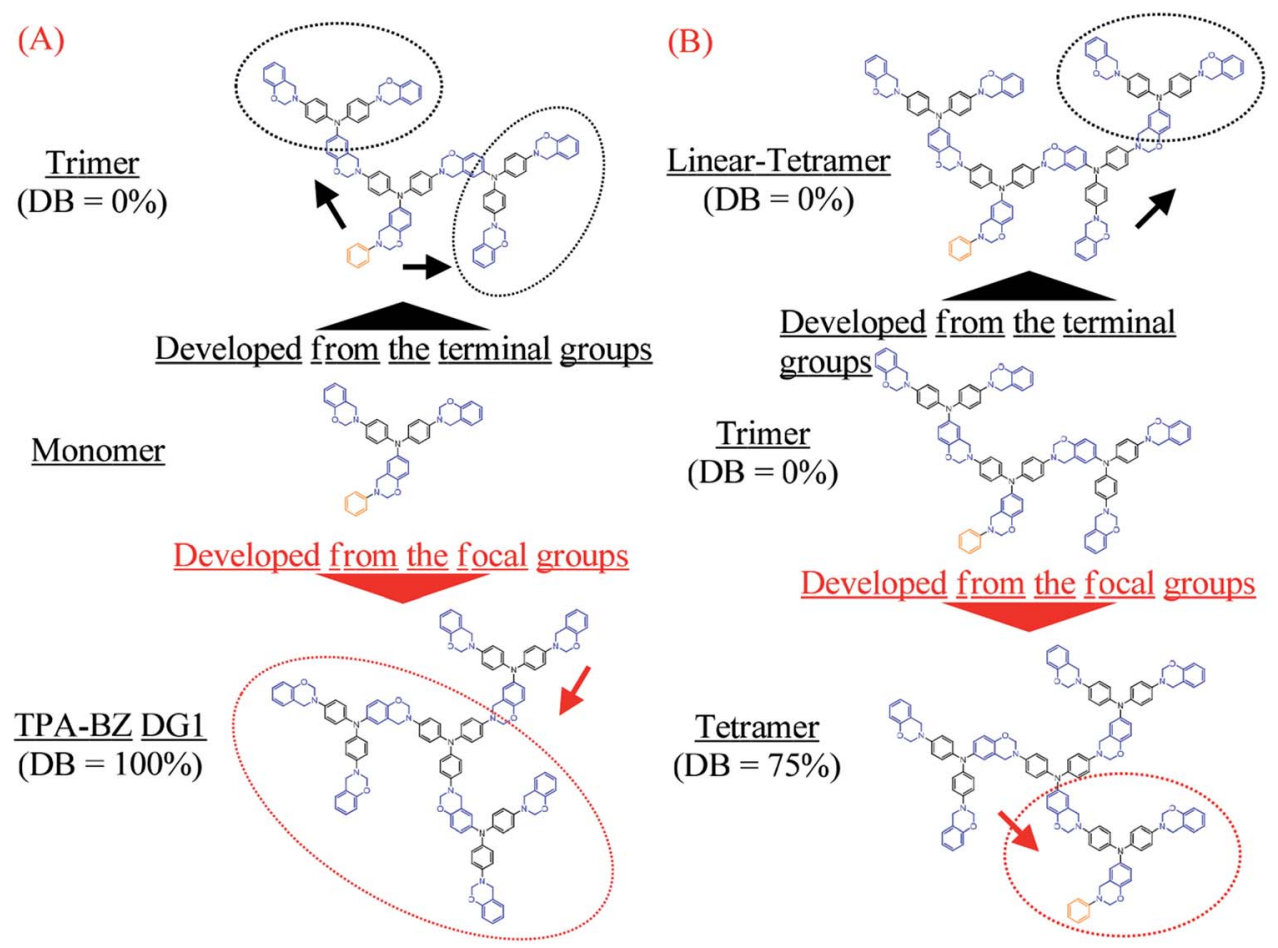

Scheme 2 Directions of development from the terminal groups and the focal group in the chemical structures of the (A) monomer and (B) trimer.

$2 \mathrm{~h}$ under reflux, TPA-2 $\mathrm{NH}_{2}-\mathrm{OH}(0.250 \mathrm{~g}, 0.858 \mathrm{mmol})$ was added. After $24 \mathrm{~h}$ under reflux, $\mathrm{CH}_{2} \mathrm{O}(0.103 \mathrm{~g}, 3.43 \mathrm{mmol})$ was added. After $2 \mathrm{~h}$ under reflux, phenol $(0.162 \mathrm{~g}, 1.72 \mathrm{mmol})$ was added. After $24 \mathrm{~h}$ under reflux, the solution was cooled to room temperature and filtered. The filtrate was concentrated under reduced pressure and the residue taken up into $\mathrm{CH}_{2} \mathrm{Cl}_{2}(3 \times 80$ $\mathrm{mL}$ ). The organic solution was washed several times with saturated aqueous $\mathrm{NaHCO}_{3}$ and distilled water, dried $\left(\mathrm{MgSO}_{4}\right.$, $30 \mathrm{~min}$ ), and concentrated. The residue was purified through column chromatography $\left(\mathrm{SiO}_{2} ; \mathrm{CH}_{2} \mathrm{Cl}_{2} / \mathrm{MeOH}, 1: 2\right)$ to yield a brown product $(0.56 \mathrm{~g}, 67 \%)$ : FTIR $\left(\mathrm{KBr}, \mathrm{cm}^{-1}\right): 1501,1337$, 1227, 941. ${ }^{1} \mathrm{H}$ NMR (500 MHz, DMSO- $d_{6}, \delta$, ppm): 6.45-7.33 (m, aromatic $\mathrm{CH}), 5.37$ and $5.44\left(\mathrm{~s}, 2 \mathrm{H}, \mathrm{OCH}_{2} \mathrm{~N}\right), 4.59$ and $4.65(\mathrm{~s}$, $\left.2 \mathrm{H}, \mathrm{CCH}_{2} \mathrm{~N}\right) .{ }^{13} \mathrm{C}$ NMR $\left(125 \mathrm{MHz}, \mathrm{DMSO}-d_{6}, \delta, \mathrm{ppm}\right): 153.91$ (peak a), 151.11 (peak b), 147.79 (peak c), 143.61 (peak d), 115.21-153.91 (aromatic), 78.64 and $79.18\left(\mathrm{OCH}_{2} \mathrm{~N}\right), 48.87$ and $49.37\left(\mathrm{CCH}_{2} \mathrm{~N}\right)$.

\section{TPA-BZ DG1}

This compound was prepared using an approach described previously. ${ }^{41}$ A solution of TPA- $3 \mathrm{NH}_{2}(0.130 \mathrm{~g}, 0.460 \mathrm{mmol})$ and $\mathrm{CH}_{2} \mathrm{O}(0.0820 \mathrm{~g}, 2.75 \mathrm{mmol})$ in 1,4-dioxane $(30 \mathrm{~mL})$ was stirred under reflux at $90{ }^{\circ} \mathrm{C}$ for $2 \mathrm{~h}$ under $\mathrm{N}_{2}$ and then TPA- $2 \mathrm{NH}_{2}-\mathrm{OH}$ $(0.400 \mathrm{~g}, 1.37 \mathrm{mmol})$ was added. After $24 \mathrm{~h}$ under reflux, $\mathrm{CH}_{2} \mathrm{O}$ $(0.170 \mathrm{~g}, 5.49 \mathrm{mmol})$ was added. After $2 \mathrm{~h}$ under reflux, phenol $(0.260 \mathrm{~g}, 2.75 \mathrm{mmol})$ was added. After $24 \mathrm{~h}$ under reflux, the solution was cooled to room temperature and filtered. The filtrate was concentrated under reduced pressure and the residue taken up into $\mathrm{CH}_{2} \mathrm{Cl}_{2}(3 \times 80 \mathrm{~mL})$. The organic solution was washed several times with saturated aqueous $\mathrm{NaHCO}_{3}$ and distilled water, dried $\left(\mathrm{MgSO}_{4}, 30 \mathrm{~min}\right)$, and concentrated. The residue was purified through column chromatography $\left(\mathrm{SiO}_{2}\right.$; $\mathrm{CH}_{2} \mathrm{Cl}_{2} / \mathrm{MeOH}, 1: 2$ ) to yield a brown product (0.56 g, 63\%): FTIR $\left(\mathrm{KBr}, \mathrm{cm}^{-1}\right): 1499,1339,1225,941 .{ }^{1} \mathrm{H}$ NMR $(500 \mathrm{MHz}$, DMSO- $\left.d_{6}, \delta, \mathrm{ppm}\right): 6.50-7.22(\mathrm{~m}$, aromatic $\mathrm{CH}), 5.38(\mathrm{~s}, 2 \mathrm{H}$, $\left.\mathrm{OCH}_{2} \mathrm{~N}\right), 4.59\left(\mathrm{~s}, 2 \mathrm{H}, \mathrm{CCH}_{2} \mathrm{~N}\right) .{ }^{13} \mathrm{C}$ NMR $\left(125 \mathrm{MHz}, \mathrm{DMSO}-d_{6}, \delta\right.$, ppm): 153.93 (peak a), 151.14 (peak b), 143.63 (peak d), 115.24153.93 (aromatic), $79.22\left(\mathrm{OCH}_{2} \mathrm{~N}\right), 49.40\left(\mathrm{CCH}_{2} \mathrm{~N}\right)$.

\section{Thermal curing of hyperbranched TPA-BZ polymers}

Desired amounts of the hyperbranched TPA-BZs were placed in Teflon dishes and thermally polymerized in a stepwise manner at $150,180,210$, and $240{ }^{\circ} \mathrm{C}$ for $2 \mathrm{~h}$ each. Each cured sample had a brown-red color that became darker upon increasing the curing temperature.

\section{Characterization}

${ }^{1} \mathrm{H}$ and ${ }^{13} \mathrm{C}$ NMR spectra were recorded using an INOVA 500 instrument with DMSO- $d_{6}$ or $\mathrm{CDCl}_{3}$ as the solvent and tetramethylsilane as the external standard. FTIR spectra of the samples were recorded using a Bruker Tensor 27 FTIR spectrophotometer; 32 scans were collected at a spectral resolution of $4 \mathrm{~cm}^{-1}$. The prepared TPA-BZ sample films were sufficiently thin to obey the Beer-Lambert law. Mass spectra were recorded using a Bruker Daltonics Autoflex matrix-assisted laser desorption ionization-time of flight (MALDI-TOF) mass spectrometer. The following voltage parameter was used: ion source 1, $19.06 \mathrm{kV}$; ion source 2, $16.61 \mathrm{kV}$; lens, $8.78 \mathrm{kV}$; reflector 1 , 
$21.08 \mathrm{kV}$; reflector 2, $9.73 \mathrm{kV}$. Dynamic curing kinetics were measured using a TA Q-20 differential scanning calorimeter operated under a $\mathrm{N}_{2}$ atmosphere. The TPA-BZ samples were placed into sealed aluminum sample pans. Dynamic curing scans were recorded from 25 to $320{ }^{\circ} \mathrm{C}$ at a heating rate of $20{ }^{\circ} \mathrm{C} \mathrm{min}^{-1}$. The thermal stability of the TPA-BZ samples was determined using a TA Q-50 thermogravimetric analyzer. A thermally cured TPA-BZ sample was placed in a Pt cell and heated at a rate of $20{ }^{\circ} \mathrm{C} \mathrm{min}{ }^{-1}$ from 25 to $800{ }^{\circ} \mathrm{C}$ under a $\mathrm{N}_{2}$ atmosphere. UV-Vis spectra were recorded using a Shimadzu mini 1240 spectrophotometer; the concentration of the hyperbranched TPA-BZ (or TPA) in THF was $10^{-4}$ M. Photoluminescence (PL) excitation and emission spectra were recorded using a monochromatized Xe light source at room temperature.

\section{Results and discussion}

\section{Preparation of four types of hyperbranched TPA-BZs and TPA-BZ DG1}

Four well-defined hyperbranched TPA-BZs-TPA-BZ monomer, TPA-BZ trimer, linear TPA-BZ tetramer, and TPA-BZ tetramer (Scheme 1 and $\mathrm{S} 1$ ) - were synthesized from aniline (as the focal group), TPA-2 $\mathrm{NH}_{2}-\mathrm{OH}$ (as the $\mathrm{AB}_{2}$ branching group), $\mathrm{CH}_{2} \mathrm{O}$, and phenol in 1,4-dioxane through facile one-pot Mannich condensations with unique feeding sequences. The facile onepot Mannich condensations employed formaldehyde/amine derivatives with uniquely activated terminal groups, rather than a strategy involving complicated and tedious protection/ deprotection of functional groups. ${ }^{41}$ The mechanism of these Mannich condensations involved three steps (Scheme S2 $\dagger$ ); the first step was the reaction of a primary amine and $\mathrm{CH}_{2} \mathrm{O}$ to form a formaldehyde/amine derivative that could be used to activate the terminal groups of the primary amino groups of the TPA-BZ dendrimers or the hyperbranched TPA-BZs; the second and third steps were the direct reactions of the formaldehyde/amine derivatives with the phenol units from phenolic derivatives to form the $\mathrm{BZ}$ ring units; these phenolic derivatives include the $\mathrm{AB}_{2}$ branching groups of TPA- $2 \mathrm{NH}_{2}-\mathrm{OH}$ and the terminal groups of phenol. ${ }^{\mathbf{4 9 , 5 0}}$ We used the unique feeding sequences of these Mannich condensations to control the chemical constructions of the TPA-BZs with various DBs. Scheme $33 \uparrow$ presents the possible mechanism of TPA-BZ trimer preparation, revealing how the feeding sequence of the reactants (aniline, TPA-2 $\mathrm{NH}_{2}-\mathrm{OH}, \mathrm{CH}_{2} \mathrm{O}$, and phenol) was very important and strongly affected the chemical construction of the TPA-BZ; the concept is somewhat similar to building a structure from bricks of LEGO. The reactions of the formaldehyde/amine derivatives (e.g., the reactions of the activated terminal groups of the hyperbranched TPA-BZs with phenolic derivatives such as TPA-2NH $\mathrm{N}_{2}-\mathrm{OH}$ ) in the first step of the Mannich condensation were "click-like" reactions (Scheme $\mathrm{S} 2 \dagger$ ). Three "click-like" reactions were used to prepare the TPA-BZ trimer (Scheme S3†); the first "click-like" reaction of the formaldehyde/amine derivative of aniline and the phenolic derivative of TPA- $2 \mathrm{NH}_{2}-\mathrm{OH}$ is presented in Scheme S3(a); $\uparrow$ the secondary and third are presented in Schemes S3(b) and S3(c), $\dagger$ respectively. The feeding sequence of the reactants could be used to control the chemical construction of the TPA-BZs through these "click-like" Mannich condensations. The feeding sequences for the preparation of the hyperbranched TPA-BZs are presented in Scheme 1 and S1. $\dagger$ TPA-BZ DG1 [Scheme 1(c)] was synthesized following an approach we have described previously. ${ }^{41}$

In our design strategy, the hyperbranched TPA-BZs were developed in the direction from the focal group or from the terminal groups of TPA-BZ monomer and TPA-BZ trimer (Scheme 2), respectively. Scheme 2A presents the two different directions for the chemical development of the TPA-BZ monomer-from its focal group to form the symmetrical TPA-BZ DG1 (downward) or from its terminal groups to form the asymmetrical TPA-BZ trimer (upward). Scheme $2 \mathrm{~B}$ presents the two different directions for the chemical development of the TPABZ trimer-from its focal group to form the asymmetrical TPABZ tetramer (downward) or from its terminal groups to form the asymmetrical linear TPA-BZ tetramer (upward). ${ }^{1} \mathrm{H}$ and ${ }^{13} \mathrm{C}$ NMR, FTIR, and MALDI-TOF mass spectra ${ }^{51-53}$ were employed to investigate the chemical constructions and properties of the hyperbranched TPA-BZs.

\section{${ }^{1}$ H NMR spectral analysis of hyperbranched TPA-BZs and TPA-BZ DG1}

Fig. 1 presents the ${ }^{1} \mathrm{H}$ NMR spectra of the hyperbranched TPABZs and TPA-BZ DG1 in DMSO- $d_{6}$ (the chemical constructions of the TPA-BZ trimer and TPA-BZ tetramer are displayed as Scheme S4 $\dagger$ ). Fig. 1a presents the significant splits of the characteristic signals of the $\mathrm{OCH}_{2} \mathrm{~N}$ groups (5.28-5.53 ppm) for the TPA-BZ monomer; more than three peaks appeared that could be grouped into two portions, because of the asymmetrical chemical construction. The signals of the $\mathrm{CCH}_{2} \mathrm{~N}$ groups (4.52-4.70 ppm) were similar to those of the $\mathbf{O C H}_{2} \mathrm{~N}$ groups. Endo et al. reported that the number of characteristics signals (oxazine rings) in the ${ }^{1} \mathrm{H}$ NMR spectra was consistent with the number of BZ groups for linear asymmetrical BZ monomers; these signal also tend to group into two portions, especially when more than three BZ units were present (namely BZ3 and BZ4). ${ }^{45}$ According to the number of signals (oxazine rings), the TPA-BZ monomer contained more than three BZ units (the theoretical number of the $\mathrm{BZ}$ units of TPA-BZ monomer), indicating that the signal splitting of the TPA-BZ monomer was more complicated than that of a linear asymmetrical monomer. The interactions of the protons of the oxazine rings and their adjacent groups were presumably influenced by the asymmetrical branching of the TPA-BZ monomer; that is, through interactions with the groups in both their main chains and branching chains.

Increasing the number of $\mathrm{BZ}$ units for the hyperbranched TPA-BZs [Fig. 1(b)-(d)] enhanced the tendency for signal splitting to two groups for both the $\mathrm{OCH}_{2} \mathrm{~N}$ and $\mathrm{CCH}_{2} \mathrm{~N}$ groups. For example, the peaks at 5.37 and $5.44 \mathrm{ppm}$ (marked $a$ and $a^{\prime}$ ) corresponding to the $\mathrm{OCH}_{2} \mathrm{~N}$ groups for TPA-BZ tetramer [Fig. 1d] featured two peaks, which we called $\mathrm{OCH}_{2} \mathrm{~N}-\mathrm{L}_{1}$ and $\mathrm{OCH}_{2} \mathrm{~N}-\mathrm{H}_{1}$, respectively. In addition, the peaks at 4.59 and 4.65 ppm (marked $b$ and $b^{\prime}$ ) correspond to $\mathrm{CCH}_{2} \mathrm{~N}$ groups; 


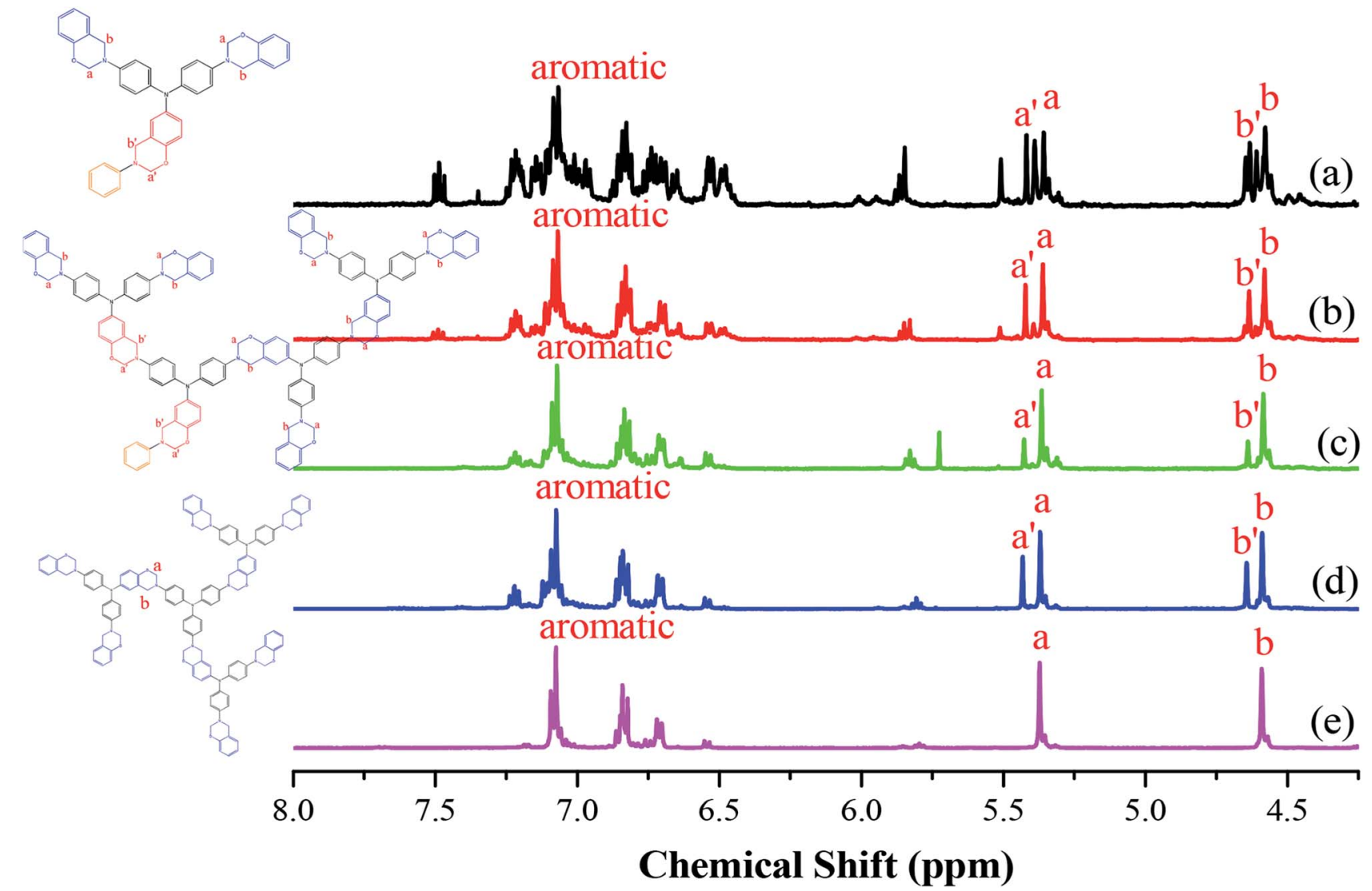

Fig. $1{ }^{1} \mathrm{H}$ NMR spectra of the TPA-BZs: (a) monomer, (b) trimer, (c) linear-tetramer, (d) tetramer, and (e) TPA-BZ DG1.

herein, these two peaks are called $\mathrm{CCH}_{2} \mathrm{~N}-\mathrm{L}_{2}$ and $\mathrm{CCH}_{2} \mathrm{~N}-\mathrm{H}_{2}$, respectively. For the oxazine rings, the tendency for signal splitting into two portions for TPA-BZ tetramer was greater than that for TPA-BZ monomer. ${ }^{45}$ In contrast to the situation for the asymmetrical TPA-BZ tetramer, the ${ }^{1} \mathrm{H}$ NMR spectrum of the symmetrical TPA-BZ DG1 (Fig. 1e) featured no signal splitting for the oxazine rings, with only two sharp signals appearing at 5.38 and $4.59 \mathrm{ppm}$ (marked $a$ and $b$ ) for the $\mathrm{OCH}_{2} \mathrm{~N}\left(\mathrm{OCH}_{2} \mathrm{~N}-\mathrm{L}_{1}\right)$ and $\mathrm{CCH}_{2} \mathrm{~N}\left(\mathrm{CCH}_{2} \mathrm{~N}-\mathrm{L}_{2}\right)$ units, respectively. These signal splits for the oxazine rings (marked $a^{\prime}$ and $b^{\prime}$ ) of the hyperbranched TPA-BZs were, therefore, related to their asymmetrical chemical constructions. Gu et al. proposed that an asymmetrical monomer containing two BZ units would feature a couple of characteristic signals in the ${ }^{1} \mathrm{H}$ NMR spectrum for both the $\mathbf{C C H}_{2} \mathrm{~N}$ and $\mathbf{O C H}_{2} \mathbf{N}$ groups. These signal are split as a result of the asymmetrical chemical construction. ${ }^{44}$ Endo et al. also reported that the ${ }^{1} \mathrm{H}$ and ${ }^{13} \mathrm{C}$ NMR spectra of a series of linear asymmetrical monomers would feature a diversity of characteristic signal splits for their oxazine rings depending on their various numbers of $\mathrm{BZ}$ units. ${ }^{45}$ As a result, the signal splits of oxazine rings can be affected by both the asymmetrical chemical constructions and the number of $\mathrm{BZ}$ units of the monomers.

Fig. 2A presents the chemical shifts of the signals for $\mathrm{OCH}_{2} \mathrm{~N}-\mathrm{H}_{1}$ and $\mathrm{OCH}_{2} \mathrm{~N}-\mathrm{L}_{1}$ in the ${ }^{1} \mathrm{H}$ NMR spectra of the hyperbranched TPA-BZs and TPA-BZ DG1. Both chemical shifts increased upon proceeding from small to large numbers of TPA
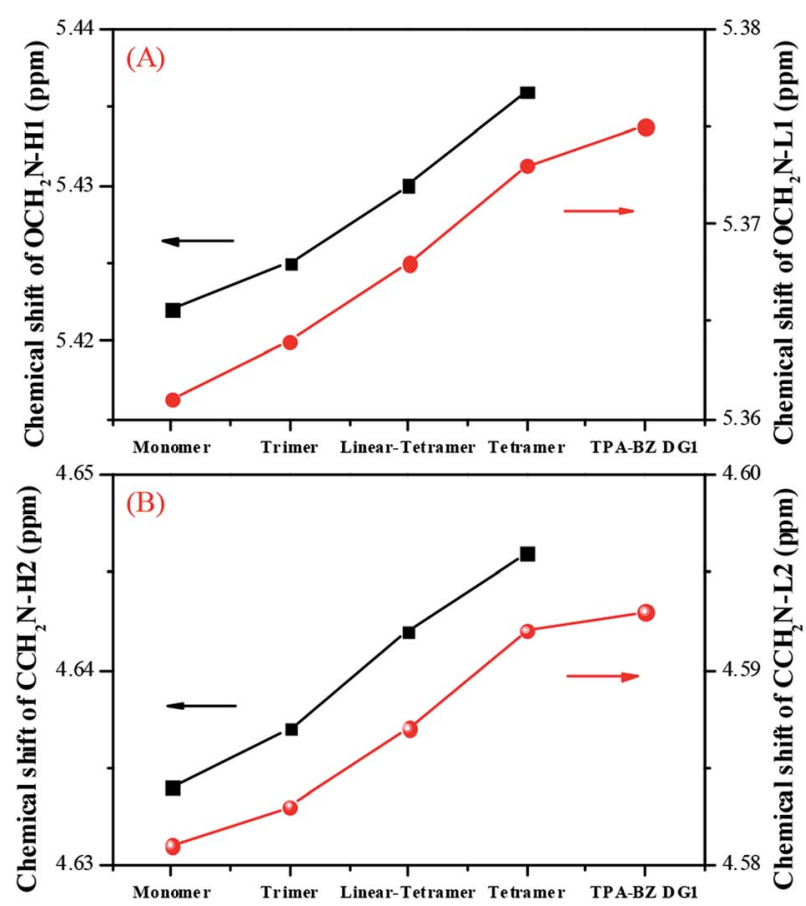

Fig. 2 Chemical shifts in ${ }^{1} \mathrm{H}$ NMR spectra of hyperbranched TPA-BZs and TPA-BZ DG1: (A) $\mathrm{OCH}_{2} \mathrm{~N}-\mathrm{H} 1$ and $\mathrm{OCH}_{2} \mathrm{~N}-\mathrm{L} 1$; (B) $\mathrm{CCH}_{2} \mathrm{~N}-\mathrm{H} 2$ and $\mathrm{CCH}_{2} \mathrm{~N}-\mathrm{L} 2$. 
Table 1 Calculation results of $H_{\mathrm{BZ}}$ and $L_{\mathrm{BZ}}$ in ${ }^{1} \mathrm{H}$ NMR spectra for hyperbranched TPA-BZs and TPA-BZ DG1 ${ }^{a}$

\begin{tabular}{|c|c|c|c|c|c|c|}
\hline Sample & & Monomer & Trimer & Linear-tetramer & Tetramer & TPA-BZ DG1 \\
\hline \multirow{2}{*}{$\mathrm{OCH}_{2} \mathrm{~N}-\mathrm{H} 1$} & $\delta, \mathrm{ppm}$ & 5.422 & 5.425 & 5.43 & 5.436 & - \\
\hline & Area (\%) & 37.2 & 41.3 & 22.0 & 34.5 & 0 \\
\hline & Area (\%) & 62.8 & 58.7 & 78.0 & 65.5 & 100.0 \\
\hline \multirow[t]{2}{*}{$\mathrm{CCH}_{2} \mathrm{~N}-\mathrm{H} 2$} & $\delta, \mathrm{ppm}$ & 4.634 & 4.637 & 4.642 & 4.646 & - \\
\hline & Area (\%) & 33.1 & 42.0 & 22.7 & 31.8 & 0 \\
\hline \multirow[t]{3}{*}{ BZ grouping } & BZ number & $3^{*}$ & $7 *$ & $9^{*}$ & $9^{*}$ & $9^{*}$ \\
\hline & $H_{\mathrm{BZ}}$ & $1.05\left(1^{*}\right)$ & $2.92\left(3^{*}\right)$ & $2.01(2 *)$ & $2.98\left(3^{*}\right)$ & $0.00\left(0^{*}\right)$ \\
\hline & $L_{\mathrm{BZ}}$ & $1.95(2 *)$ & $4.08\left(4^{*}\right)$ & $6.99\left(7^{*}\right)$ & $6.02\left(6^{*}\right)$ & $100.00(9 *)$ \\
\hline
\end{tabular}

units and DBs: TPA-BZ monomer, TPA-BZ trimer, linear TPABZ tetramer, TPA-BZ tetramer, and TPA-BZ DG1. Fig. 2B presents the chemical shifts of the signals for $\mathrm{CCH}_{2} \mathrm{~N}-\mathrm{H}_{2}$ and $\mathrm{CCH}_{2} \mathrm{~N}-\mathrm{L}_{2}$ in the ${ }^{1} \mathrm{H}$ NMR spectra of the hyperbranched TPABZs and TPA-BZ DG1; the results are similar to those in Fig. 2A. Hence, we conclude that these chemical shifts $\left(\mathrm{OCH}_{2} \mathrm{~N}-\mathrm{H}_{1}\right.$, $\mathrm{OCH}_{2} \mathrm{~N}-\mathrm{L}_{1}, \mathrm{CCH}_{2} \mathrm{~N}-\mathrm{H}_{2}$, and $\mathrm{CCH}_{2} \mathrm{~N}-\mathrm{L}_{2}$ ) are enhanced upon increasing the number of TPA units and DBs of the TPA-BZ monomers. For the signals of the oxazine rings in the ${ }^{1} \mathrm{H}$ NMR spectra, Table 1 summarizes the chemical shifts and the proportions of integrated areas (signal splits) for the hyperbranched TPA-BZs and TPA-BZ DG1.

The asymmetrical linear BZ monomer feature a number of intramolecular cross-interactions among its oxazine moieties and aromatic hydrogen atoms, thereby affecting the proton resonances of the oxazine rings, resulting in their signal splitting in ${ }^{1} \mathrm{H}$ NMR spectra; for example, the signals of the oxazine moiety connected directly to the focal group (benzyl group) tended to be located downfield in the ${ }^{1} \mathrm{H}$ NMR spectrum, comparing with those of the other oxazine rings in the monomer. ${ }^{45}$ The chemical shifts of oxazine rings depend on their relative locations in the asymmetrical $\mathrm{BZ}$ monomers; they tended to merge into two significant portions, representing the two groups of different proton resonances of the oxazine rings, as the sample concentration increased, with the resulting aggregation enhancing the intramolecular cross-interactions ${ }^{45}$ by shortening the distances the between oxazine moieties and the aromatic hydrogen atoms. The BZ units in the TPA-BZ monomers served connection or terminal functions; they all existed in the branching chemical constructions (the $\mathrm{AB}_{2}$ branching groups) and were very close to their adjacent groups, especially in those containing higher numbers of TPA and BZ units, potentially enhancing the intramolecular cross-interactions and the separation into two significant portions. The integrated areas of these two portions could be used to analyze the relationships among the characteristic signal splits and the chemical constructions of the TPA-BZ monomers.

The ratios of the integrated areas of the $\mathrm{OCH}_{2} \mathrm{~N} / \mathrm{CCH}_{2} \mathrm{~N}$ signals (oxazine rings) in the ${ }^{1} \mathrm{H}$ NMR spectra of TPA-BZ monomer, TPA-BZ trimer, linear TPA-BZ tetramer, TPA-BZ tetramer, and TPA-BZ DG1 were 2.39/2.63, 1.84/2.00, 2.00/2.11,
2.00/2.17, and 2.00/2.09, respectively; these values are all close to 1.00 , suggesting high purity for the hyperbranched TPA-BZs. Table 1 presents the integrated area proportions of the two portions of signals for the $\mathrm{OCH}_{2} \mathrm{~N}$ and $\mathrm{CCH}_{2} \mathrm{~N}$ groups; for example, the integrated area proportions of $\mathrm{OCH}_{2} \mathrm{~N}-\mathrm{H}_{1}$ and $\mathrm{OCH}_{2} \mathrm{~N}-\mathrm{L}_{1}$ for TPA-BZ tetramer were 34.5 and $65.5 \%$, respectively, while those of $\mathrm{CCH}_{2} \mathrm{~N}-\mathrm{H}_{2}$ and $\mathrm{CCH}_{2} \mathrm{~N}-\mathrm{L}_{2}$ were 31.8 and $68.2 \%$, respectively. The integrated area proportions for $\mathrm{OCH}_{2} \mathrm{~N}-\mathrm{H}_{1}$ and $\mathrm{CCH}_{2} \mathrm{~N}-\mathrm{H}_{1}$ were very close. Therefore, the number of BZ units providing downfield $\left(H_{\mathrm{BZ}}\right)$ signals in the ${ }^{1} \mathrm{H}$ NMR spectra could be calculated using the equation (theoretical number of $\mathrm{BZ}$ units of the hyperbranched TPA-BZs) $\times$ (average integrated area proportion of $\mathrm{OCH}_{2} \mathrm{~N}-\mathrm{H}_{1}$ and $\mathrm{CCH}_{2} \mathrm{~N}-\mathrm{H}_{1}$ for the hyperbranched TPA-BZs)/100. The number of $\mathrm{BZ}$ groups providing upfield $\left(L_{\mathrm{BZ}}\right)$ signals in the ${ }^{1} \mathrm{H}$ NMR spectra was calculated using the equation (theoretical number of $\mathrm{BZ}$ units of the hyperbranched TPA-BZs) - $\left(H_{\mathrm{BZ}}\right.$ of the hyperbranched TPABZs). For example, the value of $H_{\mathrm{BZ}}$ of TPA-BZ tetramer was 2.98 $=[9 \times(34.5+31.8) / 2 / 100]$; the value of $L_{\mathrm{BZ}}$ of TPA-BZ tetramer was $6.02=[9-2.98]$. These values for TPA-BZ tetramer are very close to three and six, respectively, suggesting that there were three BZ units $\left(H_{\mathrm{BZ}}=3\right)$ that were possibly affected by the asymmetrical chemical construction and that their signal splits were located downfield in the ${ }^{1} \mathrm{H}$ NMR spectra, comparing with those of $L_{\mathrm{BZ}}$. These three $\mathrm{BZ}$ units were presumably positioned in some unique locations in the chemical constructions of TPABZ tetramer. Such $H_{\mathrm{BZ}}$ analysis can possibly be used to investigate the chemical constructions of hyperbranched TPA-BZs. Table 1 presents the calculated number of BZs, and the values of $H_{\mathrm{BZ}}$ and $L_{\mathrm{BZ}}$, in the ${ }^{1} \mathrm{H}$ NMR spectra of the hyperbranched TPABZs.

The characteristic signal splits (oxazine rings) in the ${ }^{1} \mathrm{H}$ NMR spectra for the linear asymmetrical monomers resulted from the protons of the oxazine rings interacting with adjacent aromatic hydrogen atoms, based on an analysis using ${ }^{1} \mathrm{H}^{-1} \mathrm{H}$ NOESY 2D NMR spectroscopy, because the chemical constructions of the monomer featured a BZ ring group with a diversity of connected components at its two ends: including BZ rings or a benzyl group (the focal group) or empty (a hydrogen atom). ${ }^{45}$ The chemical constructions of the hyperbranched TPA-BZs or TPA-BZ DG1 featured a $>N$-(benzyl-BZ)- $N<$ unit, which had 
a benzyl BZ group (b-BZ set) with a diversity of connected components at its two ends (e.g., the nitrogen atoms with their connected b-BZ sets), depending on the role served by the b-BZ set in the TPA-BZ monomer (e.g., focal, connection, or terminal function). Therefore, the characteristic signal splits (oxazine rings) of the b-BZ set in the ${ }^{1} \mathrm{H}$ NMR spectra of hyperbranched TPA-BZs and TPA-BZ DG1 were possibly affected by the adjacent b-BZ sets connected to the nitrogen atoms at its two ends (intramolecular cross-interactions); thus, it might possibly be used to analyze the relationships between the signal splitting and the chemical constructions of the hyperbranched TPA-BZs.

Consequently, we selected a piece of the structural composition, which possessed a b-BZ set with its two ends connected to components (e.g., the nitrogen atoms and their connected b$\mathrm{BZ}$ sets), to analyze the proton resonances of the oxazine rings in the ${ }^{1} \mathrm{H}$ NMR spectra ( $H_{\mathrm{BZ}}$ and $L_{\mathrm{BZ}}$ portions) for the TPA-BZs. The structural compositions of the hyperbranched TPA-BZs and the TPA-BZ DG1 were analyzed and sorted in terms of the roles served by the $\mathrm{b}-\mathrm{BZ}$ and its two end-connected compositions (composed by several b-BZ sets and nitrogen atoms) components (Table S1†). The code rules of the structural in this study are presented in Tables $\mathrm{S} 1$ and $\mathrm{S} 4: \dagger$ (i) the arrow in red indicates the position of the designated $\mathrm{BZ}$ ring $\left({ }^{1} \mathrm{H}\right.$ NMR spectra) or the designated carbon atom $\left({ }^{13} \mathrm{C}\right.$ NMR spectra); (ii) the numbers $1,2, \underline{2}$, and 3 represent the $\mathrm{b}-\mathrm{BZ}$ sets serving the focal function, the connection function, the connection function featuring a connected component of a core group of the TPA-BZ dendrimer, and the terminal function, respectively; (iii) the focal composition of $[F x x]$ : " $F$ " indicates the focal composition and " $x x$ " indicates that the b-BZ set of the focal function has two b-BZ sets at its end nitrogen atom close to the BZ ring (e.g.) [F33] indicates that one focal function set has two terminal function sets at its end nitrogen atom closed to the BZ ring); (iv) the connection composition of [Cxxyy]: " $C$ " indicates the connection composition, " $x x$ " indicates that the b-BZ set of the connection function has two b-BZ sets at its end nitrogen atom close to the $\mathrm{BZ}$ ring, and " $y \mathrm{y}$ " indicates that the b-BZ set of the connection function has two b-BZ sets at its end nitrogen atom close to the benzyl group (e.g., [C3312] indicates that one connection function set has two terminal function sets at its end nitrogen atom close to the BZ ring, and one focal and one connection function set at its end nitrogen atom close to the benzyl group); (v) the terminal composition of $[T x x]$ : " $T$ " indicates the terminal composition and " $x x$ " indicates that the b-BZ set of the terminal function has two b-BZ sets at its end nitrogen atom close to the benzyl group (e.g., [T12] indicates that one terminal function set has one focal and one connection function set at its end nitrogen atom close to the benzyl group).

We used 14 types of structural compositions (Table S1 $\dagger$ ) to analyze the signals of $H_{\mathrm{BZ}}$ and $L_{\mathrm{BZ}}$ in the ${ }^{1} \mathrm{H}$ NMR spectra of the hyperbranched TPA-BZs and TPA-BZ DG1; for example, TPA$\mathrm{BZ}$ trimer was composed of three types of structural compositions: one piece of [F22], two pieces of [C3312], and four pieces of [T23]. These structural compositions could be used to analyze the relationship between the chemical construction and the portions of $L_{\mathrm{BZ}}$ and $H_{\mathrm{BZ}}$, which represented the characteristic signal splits of the oxazine rings in the downfield region of the
${ }^{1} \mathrm{H}$ NMR spectra. We employed a systematic methodology using the 14 types of structural compositions to analyze the relationship between the $H_{\mathrm{BZ}}$ portion and the chemical constructions of the hyperbranched TPA-BZs and TPA-BZ DG1. Table S1 $\dagger$ reveals the six types of structural compositions possibly belonging to the characteristic signal splits of the oxazine rings in the downfield region of the ${ }^{1} \mathrm{H}$ NMR spectra (marked $H_{\mathrm{BZ}}$ ): [F33], [F23], [F22], [C3312], [C2213], and [T12]. In other words, the chemical constructions of TPA-BZ monomers possessing these six types of structural compositions would possibly lead to the characteristic signal splits (oxazine rings) observed in the downfield region of the ${ }^{1} \mathrm{H}$ NMR spectra; for example, there are three types of structural compositions of TPA-BZ tetramer belonging to the $H_{\mathrm{BZ}}$ portion $\left(H_{\mathrm{BZ}}=3\right)$ : one piece of [F23], one piece of [C2213], and one piece of [T12]. The analytical results for the values of $H_{\mathrm{BZ}}$ and $L_{\mathrm{BZ}}$ in Table $\mathrm{S} 1 \dagger$ are consistent with the calculated results determined from the ${ }^{1} \mathrm{H}$ NMR spectra in Table 1. These six types of structural compositions could be used to analyze the relationship between the $H_{\mathrm{BZ}}$ portion in the ${ }^{1} \mathrm{H}$ NMR spectra and the chemical constructions of the hyperbranched TPA-BZs and TPA-BZ dendrimers.

Scheme 3 presents the possible mechanism of $H_{\mathrm{BZ}}$ and $L_{\mathrm{BZ}}$ portion analysis for the hyperbranched TPA-BZs and TPA-BZ DG1; the BZ rings in red and blue belong to the $H_{\mathrm{BZ}}$ and $L_{\mathrm{BZ}}$ portions, respectively, in the ${ }^{1} \mathrm{H}$ NMR spectra. The values of $H_{\mathrm{BZ}}$ and $L_{\mathrm{BZ}}$ are summarized in Table 2. For hyperbranched TPABZs, the b-BZ set of the focal function (e.g., [F33], [F23], and [F22]) and the b-BZ sets connected at its end nitrogen atom close to the BZ ring (e.g., the connection function sets of [C3312] and [C2213], and terminal function set of [T12]) belong to the $H_{\mathrm{BZ}}$ portion, except in the connection function set of [C2312] for linear TPA-BZ tetramer. As a result, the characteristic signal splits (oxazine rings) in the ${ }^{1} \mathrm{H}$ NMR spectra are affected strongly by the focal group (benzyl group). All BZ rings (all structural compositions) of TPA-BZ DG1 belong to the $L_{\mathrm{BZ}}$ portion, due to its symmetrical chemical construction. For the TPA-BZs containing four TPA groups, the $H_{\mathrm{BZ}}$ can be used to distinguish the conformations of the TPA-BZs (DBs from 0 to $100 \%$ ), even when these compounds possess the same molecular weight; for example, the values of $H_{\mathrm{BZ}}$ of linear-tetramer $(\mathrm{DB}=0 \%)$, tetramer $(\mathrm{DB}=75 \%)$, and TPA-BZ DG1 $(\mathrm{DB}=$ $100 \%$ ) were two, three, and zero, respectively (Table 2). Therefore, these six structural compositions of the $H_{\mathrm{BZ}}$ portion in the ${ }^{1} \mathrm{H}$ NMR spectra could be used as a powerful tool to investigate the chemical constructions of hyperbranched and dendrimer TPA-BZs, especially for those with higher numbers of TPA and $\mathrm{BZ}$ units and various DBs.

The design strategies of the TPA-BZ chemical constructions (Scheme 2) were also used to analyze the relationship between the value of $H_{\mathrm{BZ}}$ and the chemical construction developments of TPA-BZ monomer and TPA-BZ trimer. Scheme 3 and Table $\mathrm{S} 2 \dagger$ present the relationships between the value of $H_{\mathrm{BZ}}$ and their chemical construction developments as the number of TPA or BZ units increased for TPA-BZ monomer and TPA-BZ trimer. TPA-BZ DG1 comprised three TPA-BZ monomers developed from their focal groups connected to the same center nitrogen atom, and the value of $H_{\mathrm{BZ}}$ of TPA-BZ 


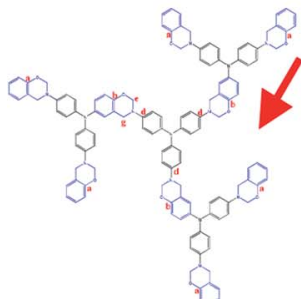

TPA-BZ DG1 $(\mathrm{DB}=100 \%)$ $\uparrow$ symmetrical
Developed from the terminal groups

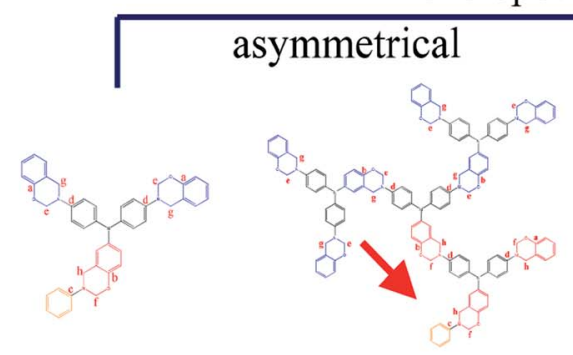

Monomer
Tetramer $(\mathrm{DB}=75 \%)$
Developed from the focal group

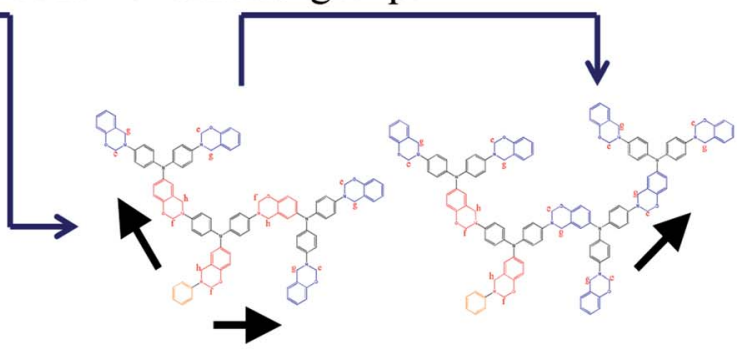

Trimer $(\mathrm{DB}=0 \%)$
Linear-Tetramer $(\mathrm{DB}=0 \%)$

Scheme 3 Possible mechanism of $H_{B Z}, L_{B Z}$, and $C_{f}$ analysis for hyperbranched TPA-BZs and TPA-BZ DG1.

Table 2 Sample information and values of $H_{B Z}, L_{B Z}$, and $C_{f}$ of the hyperbranched TPA-BZs and TPA-BZ DG1 ${ }^{a}$

\begin{tabular}{|c|c|c|c|c|c|c|}
\hline DB (\%) & & 100 & - & 75 & 0 & 0 \\
\hline Type & & Dendrimer & Hyperbranched & Hyperbranched & Hyperbranched & Hyperbranched \\
\hline BZ group & & 9 & 3 & 9 & 7 & 9 \\
\hline \multirow[t]{2}{*}{${ }^{1} \mathrm{H}$ NMR } & $H_{\mathrm{BZ}}$ & 0 & 1 & 3 & 3 & 2 \\
\hline & $L_{\mathrm{BZ}}$ & 9 & 2 & 6 & 4 & 7 \\
\hline & $P_{\mathrm{c}}$ & - & 0.029 & 0.007 & 0 & 0 \\
\hline & $P_{\mathrm{d}}$ & 0.029 & 0.029 & 0.007 & 0 & 0 \\
\hline & $P_{\mathrm{e}}$ & 0.036 & 0.029 & 0 & 0 & 0 \\
\hline & $P_{\mathrm{f}}$ & - & 0.029 & 0.007 & 0 & 0 \\
\hline & $P_{\mathrm{g}}$ & 0.037 & 0.037 & 0.007 & 0 & 0 \\
\hline & $P_{\mathrm{h}}$ & - & 0.029 & 0 & 0 & 0 \\
\hline
\end{tabular}

${ }^{a}$ The $H_{\mathrm{BZ}}, L_{\mathrm{BZ}}$, and $C_{\mathrm{f}}\left(P_{\mathrm{e}}-P_{\mathrm{h}}\right)$ belong to the characteristic signals of oxazine rings.

monomer decreased from one to zero as a result of the symmetrical dendrimer formation of TPA-BZ DG1. The linear asymmetrical TPA-BZ trimer was developed from the terminal groups of TPA-BZ monomer by adding two single $\mathrm{AB}_{2}$ branching groups of TPA with the BZ terminal groups connected to TPA-BZ monomer's two terminal groups; the value of $H_{\mathrm{BZ}}$ of TPA-BZ monomer increased from one to three. The branching asymmetrical TPA-BZ tetramer was developed from the focal group of TPA-BZ trimer, which connected to one of the terminal groups of the single $\mathrm{AB}_{2}$ branching group of TPA containing one focal group and one $\mathrm{BZ}$ terminal group; the value of $H_{\mathrm{BZ}}$ of TPA-BZ trimer decreased from three to one. The value of $H_{\mathrm{BZ}}$ of TPA-BZ tetramer, however, was also three; it belonged to the three b-BZ sets connected to the center nitrogen atom containing a b-BZ set of focal function. The linear asymmetrical linear TPA-BZ tetramer was developed from the terminal group of the trimer by adding a single $\mathrm{AB}_{2}$ branching group of TPA containing two BZ terminal groups connected to one of the terminal groups of TPA-BZ trimer; the value of $H_{\mathrm{BZ}}$ of TPA-BZ trimer decreased from three to two.

The BZ groups (the b-BZ sets) of the $H_{\mathrm{Bz}}$ portion were usually located in the connected components at the end nitrogen atom of the focal function set. Interestingly, the value of $H_{\mathrm{BZ}}$ of TPABZ trimer decreased after the addition of a TPA group connected to its one terminal group, resulting in the formation of the linear asymmetrical linear TPA-BZ tetramer. Hence, the linear asymmetrical TPA-BZ monomer $(\mathrm{DB}=0 \%)$ developed from the terminal groups of TPA-BZ trimer possibly led to the decrease in the value of $H_{\mathrm{BZ}}$. The values of $H_{\mathrm{BZ}}$ for the TPA-BZ monomers in this study ranged from zero to three; a value of zero indicates that the TPA-BZ monomer belongs to the symmetrical dendrimer; a value of $H_{\mathrm{BZ}} / L_{\mathrm{BZ}}$ of $1 / 2$ indicates that the TPA-BZ is the monomer; a value of $H_{\mathrm{BZ}} / L_{\mathrm{BZ}}$ of $2 / 7$ indicates that the TPA-BZ is the linear-tetramer; a value of $H_{\mathrm{BZ}} / L_{\mathrm{BZ}}$ of $3 / 4$ indicates that the TPA-BZ is the trimer; a value of $H_{\mathrm{BZ}} / L_{\mathrm{BZ}}$ of $3 / 6$ indicates that the TPA-BZ is the tetramer. Hence, the pairs of values of $H_{\mathrm{BZ}}$ and 
$L_{\mathrm{BZ}}$ can be used conveniently to identify the chemical constructions and conformations of the TPA-BZs.

\section{${ }^{13}$ C NMR spectral analysis of hyperbranched TPA-BZs and TPA-BZ DG1}

Fig. 3 presents the ${ }^{13} \mathrm{C}$ NMR spectra of the hyperbranched TPABZs and TPA-BZ DG1 in DMSO- $d_{6}$ (the chemical constructions of the annotated ${ }^{13} \mathrm{C}$ NMR spectra for TPA-BZ trimer and TPA$\mathrm{BZ}$ tetramer are displayed in Scheme $\mathrm{S} 5 \dagger$ ). Fig. $\mathrm{S} 2 \dagger$ provides an enlarged view of the ${ }^{13} \mathrm{C}$ NMR spectrum of TPA-BZ monomer (Fig. 3a) from 138 to 168 ppm. Fig. 3(a)-(c) present the ${ }^{13} \mathrm{C}$ NMR spectra of TPA-BZ monomer, TPA-BZ trimer, and linear TPA-BZ tetramer, respectively, including four characteristic signals in the range from 143 to $154 \mathrm{ppm}$ (peaks a-d) and four characteristic signals of oxazine rings in the range from 48 to $80 \mathrm{ppm}$ (peaks e-h), including two characteristic signal splits (peak $\mathrm{f}$ and $\mathrm{h}$ ) arising from the asymmetrical chemical constructions, similar to the situation in the ${ }^{1} \mathrm{H}$ NMR spectra. Fig. 3d presents the ${ }^{13} \mathrm{C}$ NMR spectrum of TPA-BZ tetramer; peak a (namely $P_{\mathrm{a}}$; $153.912 \mathrm{ppm}$ ) corresponds to the aromatic $\mathrm{CH}_{2} \mathrm{OC}$ nuclei of the b-BZ sets of the terminal function; peak b (namely $P_{\mathrm{b}} ; \mathbf{1 5 1 . 1 1 3}$ ppm) corresponds to the aromatic $\mathrm{CH}_{2} \mathrm{OC}$ nuclei of the b-BZ sets of the connection and focal functions; peak c (namely $P_{\mathrm{c}}$; $147.794 \mathrm{ppm}$ ) corresponds to the aromatic $\mathrm{CH}_{2} \mathrm{NC}$ nuclei of the b-BZ sets of the focal functions; peak d (namely $P_{\mathrm{d}} ; 143.611$ ppm) corresponds to the aromatic $\mathrm{CH}_{2} \mathrm{NC}$ nuclei of the b-BZ sets of connection and terminal functions; peaks e and $\mathrm{f}$ (namely $P_{\mathrm{e}}$ and $P_{\mathrm{f}} ; 79.183$ and 78.640 ppm, respectively) correspond to $\mathrm{OCH}_{2} \mathrm{~N}$ nuclei of the oxazine rings; and peaks $\mathrm{g}$ and $\mathrm{h}$ (namely $P_{\mathrm{g}}$ and $P_{\mathrm{h}} ; 49.371$ and $48.866 \mathrm{ppm}$ ) correspond to the $\mathrm{CCH}_{2} \mathrm{~N}$ nuclei of the oxazine rings. The chemical shifts of the characteristic signals in the ${ }^{13} \mathrm{C}$ NMR spectra of the hyperbranched TPA-BZs and TPA-BZ DG1 are summarized in Table S3. $\dagger$ Fig. 3e presents the ${ }^{13} \mathrm{C}$ NMR spectrum of TPA-BZ DG1 in DMSO- $d_{6} . P_{\mathrm{a}}$ and $P_{\mathrm{b}}$ (153.934 and $151.143 \mathrm{ppm}$, respectively) correspond to the aromatic $\mathrm{CH}_{2} \mathrm{OC}$ nuclei of the b-BZ sets of the terminal and the connection functions, respectively. $P_{\mathrm{d}}(143.633$ ppm) corresponds to the aromatic $\mathrm{CH}_{2} \mathrm{NC}$ nuclei of the b-BZ sets of the connection and terminal functions. $P_{\mathrm{e}}$ (79.219 ppm) corresponds to $\mathrm{OCH}_{2} \mathrm{~N}$ nuclei of the oxazine rings. $P_{\mathrm{g}}$ (49.401 ppm) corresponds to $\mathrm{CCH}_{2} \mathrm{~N}$ nuclei of the oxazine rings. TPA-BZ DG1 has no signal $P_{\mathrm{c}}$ because the dendrimer lacked a focal group (benzyl group). The spectrum of TPA-BZ DG1 features only one signal for each $\mathrm{OCH}_{2} \mathrm{~N}$ and $\mathrm{CCH}_{2} \mathrm{~N}$ nucleus $\left(P_{\mathrm{e}}\right.$ and $P_{\mathrm{g}}$, respectively). In contrast, the spectrum of TPA-BZ tetramer has two signals for each $\mathrm{OCH}_{2} \mathrm{~N}\left(P_{\mathrm{e}}\right.$ and $\left.P_{\mathrm{f}}\right)$ and $\mathrm{CCH}_{2} \mathrm{~N}$ $\left(P_{\mathrm{g}}\right.$ and $\left.P_{\mathrm{h}}\right)$ nucleus, due to its asymmetrical chemical construction. Hence, the asymmetrical chemical construction of a TPA-BZ enhances the tendency of the characteristic signal splits $\left(P_{\mathrm{f}}\right.$ and $\left.P_{\mathrm{h}}\right)$ of the oxazine rings in the upfield region of the ${ }^{13} \mathrm{C}$ NMR spectra, compared with that of their symmetrical counterparts; the behavior is similar to that of linear BZ monomers. ${ }^{45}$ Interestingly, the characteristic signal splits of the

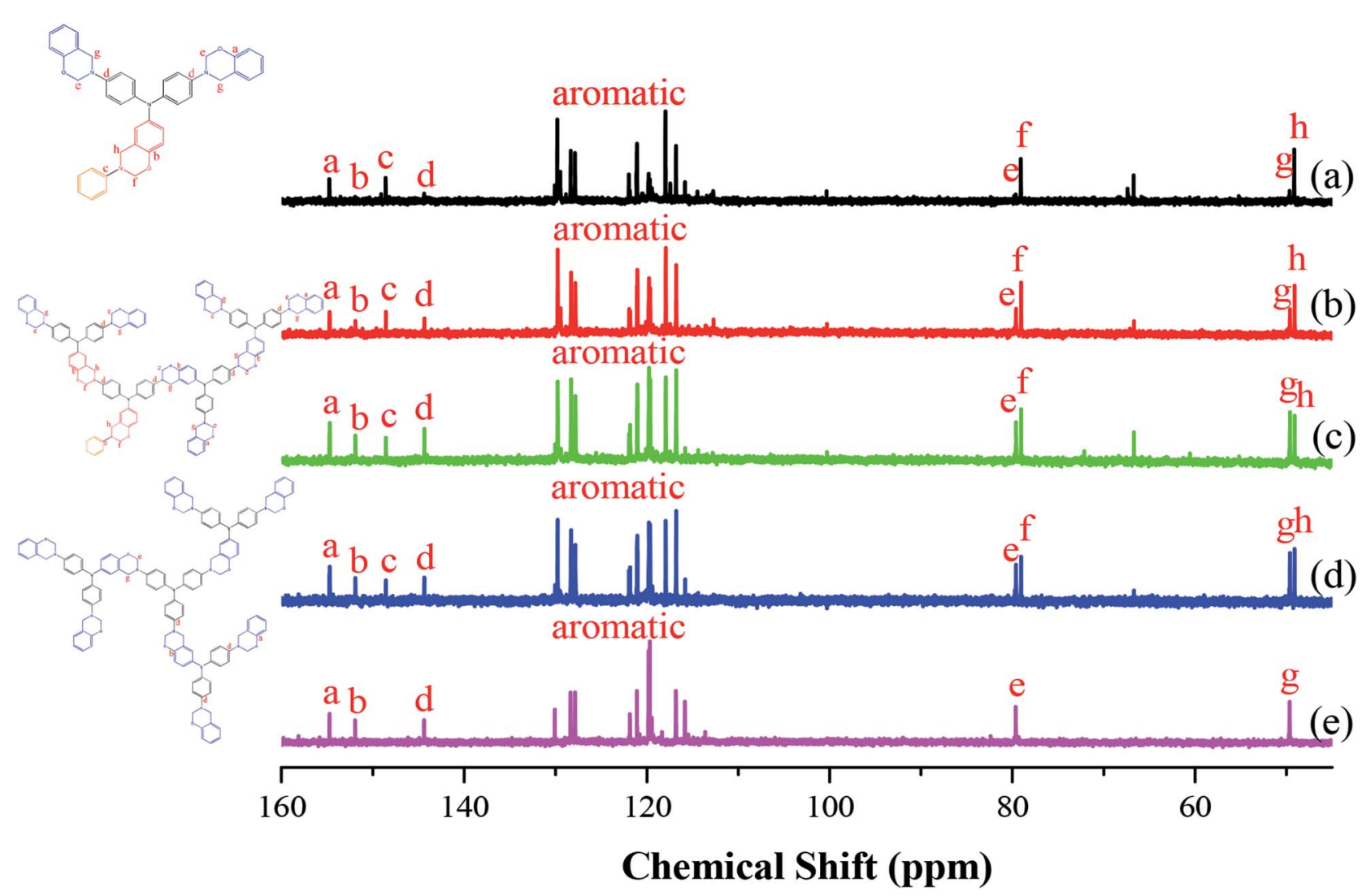

Fig. $3{ }^{13}$ C NMR spectra of the TPA-BZs: (a) monomer, (b) trimer, (c) linear-tetramer, (d) tetramer, and (e) TPA-BZ DG1. 
oxazine rings for TPA-BZs arising from the asymmetrical chemical construction appear downfield and upfield, compared with those of the symmetrical counterparts, in the ${ }^{1} \mathrm{H}$ and ${ }^{13} \mathrm{C}$ NMR spectra, respectively.

Fig. S3† presents the chemical shifts of $P_{\mathrm{a}}-P_{\mathrm{d}}$ in the ${ }^{13} \mathrm{C}$ NMR spectra of the hyperbranched TPA-BZs and TPA-BZ DG1; the distribution of these data for each peak followed a similar tendency of three steps of (i) decreasing, (ii) keeping constant, and (iii) increasing, as the sample order changed from TPA-BZ monomer, trimer, linear-tetramer, tetramer, to TPA-BZ DG1. Fig. 4 presents the chemical shifts $\mathrm{P}_{\mathrm{e}}-\mathrm{P}_{\mathrm{h}}$ of the oxazine rings in the ${ }^{13} \mathrm{C}$ NMR spectra of the hyperbranched TPA-BZs and TPABZ DG1, revealing similar data distributions of the three steps as those in Fig. $\mathrm{S} 3 \dagger\left(P_{\mathrm{a}}-P_{\mathrm{d}}\right)$; the situation is very different from that in the ${ }^{1} \mathrm{H}$ NMR spectra. Interestingly, the chemical shift of each peak $P_{\mathrm{a}}-P_{\mathrm{h}}$ for TPA-BZ trimer and linear TPA-BZ tetramer remained unchanged at a relative lower constant, comparing with those of the other three monomers in this study, indicating that these eight chemical shifts for these linear TPA-BZ monomers ( $\mathrm{DB}=0 \%$ ) were possibly not affected by the effect of the number of TPA or BZ groups.

The ${ }^{13} \mathrm{C}$ NMR spectral data for the linear asymmetrical monomers $\mathrm{BZ} 2, \mathrm{BZ} 3$, and $\mathrm{BZ}^{45}$ revealed four characteristic signal portions in the range from 142.5 to $154.4 \mathrm{ppm}$, representing the aromatic carbon nuclei of the $\mathrm{CH}_{2} \mathrm{OC}$ and $\mathrm{CH}_{2} \mathrm{NC}$ groups, and four characteristic signal portions in the range from 50.5 to $80.5 \mathrm{ppm}$, representing the $\mathrm{OCH}_{2} \mathrm{~N}$ and $\mathrm{CCH}_{2} \mathrm{~N}$ groups; the mergence extension for these eight signal portions possibly can be enhanced by using the solution concentration enhancement, resulting in the intramolecular crossinteractions increment. As a result, these eight signal portions also tended to keep at an approximate constant chemical shift for each signal portion, similar to the behavior of $P_{\mathrm{a}}-P_{\mathrm{h}}$ for the linear TPA-BZ trimer and linear-tetramer. These eight signals $\left(P_{\mathrm{a}}-P_{\mathrm{h}}\right)$ of the hyperbranched TPA-BZs exhibited well mergence extension for each signal, indicative of enhanced intramolecular cross-interactions, possibly resulting from shorter distances between the designated carbon atoms and their adjacent groups, due to their branched chemical structures $\left(\mathrm{AB}_{2}\right.$ branching groups), compared with those of the linear asymmetrical BZ monomers, similar to the behavior in the ${ }^{1} \mathrm{H}$ NMR spectra. Interestingly, these characteristic signals (portions) of the linear asymmetrical monomers for hyperbranched TPA-BZs and all tended to remain approximately constant chemical shift, and were not affected by the incorporation of the TPA groups into the monomers or the branched chemical structures; this phenomenon can, therefore, be used as a standard or base when using ${ }^{13} \mathrm{C}$ NMR spectroscopy to analyze the chemical structures or conformations of asymmetrical TPA-BZ monomers. In addition, these eight characteristic signals in the ${ }^{13} \mathrm{C}$ NMR spectra are affected strongly upon changing the relative position of the attached oxazine ring in the monomer [e.g., in the para, meta, or ortho position of the linear asymmetrical monomer solely containing two BZ moieties (BZ2)]. ${ }^{46}$ These chemical shifts $\left(P_{\mathrm{a}}-P_{\mathrm{h}}\right)$ for TPA-BZ monomer and TPA-BZ DG1 appear at relatively higher values compared with those of TPA-

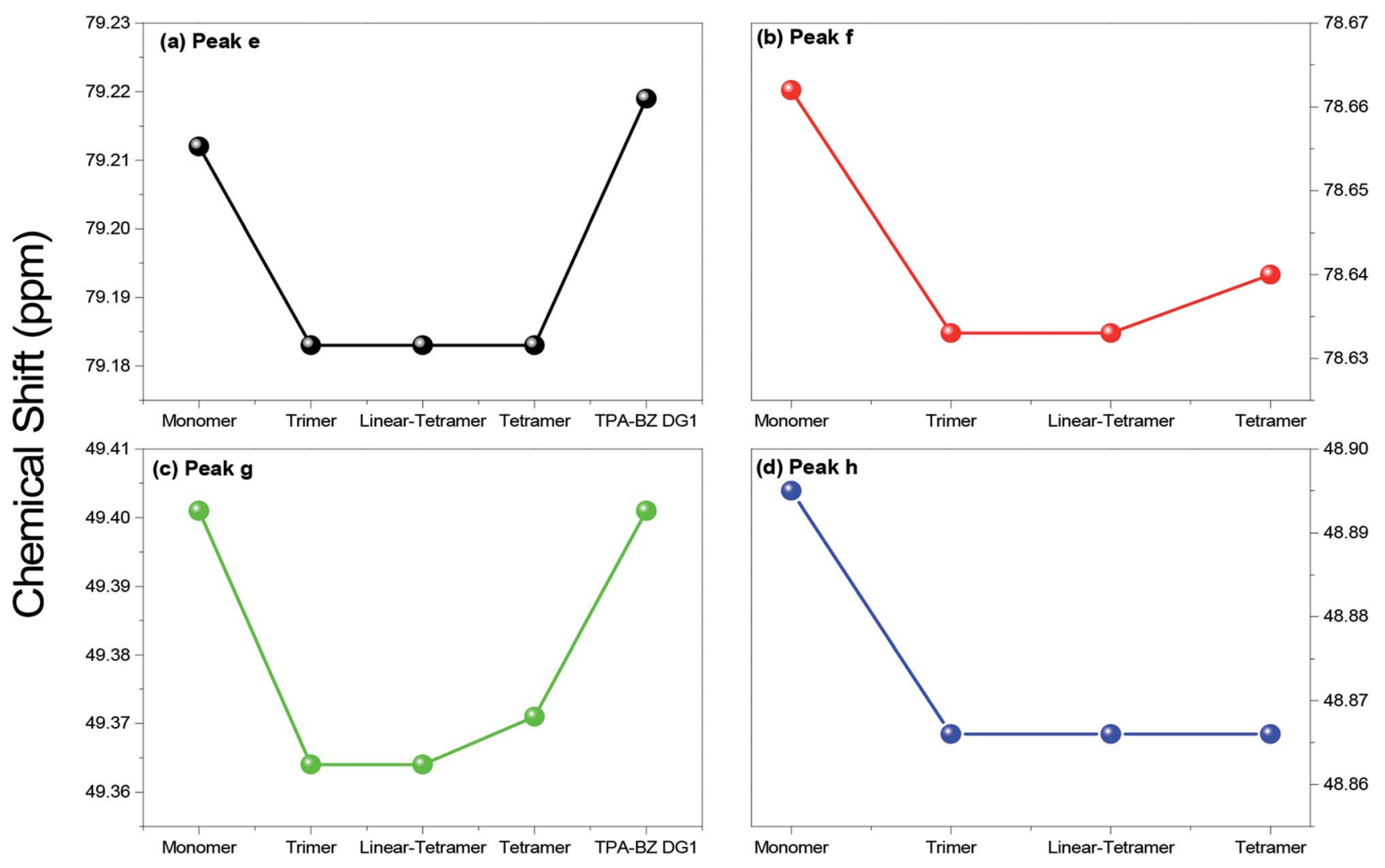

Fig. 4 Chemical shifts in ${ }^{13}$ C NMR spectra of hyperbranched TPA-BZs and TPA-BZ DG1 for peaks (a) e, (b) f, (c) g, and (d) h. 
BZ tetramer. The chemical shifts $\left(P_{\mathrm{a}}-P_{\mathrm{h}}\right)$ of TPA-BZ tetramer are located between those of TPA-BZ DG1 and linear TPA-BZ tetramer; these three monomers have the same molecular weight, but different chemical conformations (Table 2). As a result, the eight chemical shifts $\left(P_{\mathrm{a}}-P_{\mathrm{h}}\right)$ of the monomers containing four TPA groups and night BZ groups should shift downfield as the DB increases.

According to this analysis of the eight signals $P_{\mathrm{a}}-P_{\mathrm{h}}$, the linear TPA-BZs (trimer and linear-tetramer featuring $\mathrm{DB}=0 \%$ ) exhibited the most upfield chemical shifts that remained unchanged for each peak, relative to those of the other three monomers in this study. We suspect that the chemical conformations of the TPA-BZ monomers affected these chemical shifts $\left(P_{\mathrm{a}}-P_{\mathrm{h}}\right)$. The eight chemical shifts $\left(P_{\mathrm{a}}-P_{\mathrm{h}}\right)$ of lineartetramer could be used as a base for examining those of the other monomers in this study. We define the value of $C_{\mathrm{f}}(\mathrm{ppm})$ in the ${ }^{13} \mathrm{C}$ NMR spectrum for peak $x$ (namely $\mathrm{P}_{x} ; x=\mathrm{a}-\mathrm{h}$ ) as [(chemical shift of $\mathrm{P}_{x}$ of TPA-BZ monomer) - (chemical shift of $\mathrm{P}_{x}$ of linear-tetramer)]. Table 2 summarizes the values of $C_{\mathrm{f}}$ in the ${ }^{13} \mathrm{C}$ NMR spectra of the hyperbranched TPA-BZs and TPABZ DG1. The chemical shifts $\left(P_{\mathrm{a}}-P_{\mathrm{h}}\right)$ were affected by the chemical constructions and conformations, especially by the DB. Hence, the values of $C_{\mathrm{f}}$ were more suitable than the chemical shifts alone when evaluating the chemical constructions and conformations of the TPA-BZ monomers; that is, the differences in their chemical constructions could be investigated more clearly.

Fig. S4 $\dagger$ presents the values of $C_{\mathrm{f}}$ of $P_{\mathrm{a}}-P_{\mathrm{d}}$ for the hyperbranched TPA-BZs and TPA-BZ DG1. For TPA-BZ monomer, these values were higher than those of the other hyperbranched TPA-BZs, especially for $P_{\mathrm{a}}(0.058 \mathrm{ppm})$. The values of $C_{\mathrm{f}}\left(P_{\mathrm{a}}-P_{\mathrm{d}}\right)$ of TPA-BZ trimer all remained at a constant at zero, due to the linear structure of TPA-BZ monomer $(\mathrm{DB}=0 \%)$. The values of $C_{\mathrm{f}}$ for signals $P_{\mathrm{a}}, P_{\mathrm{b}}$, and $P_{\mathrm{d}}$ of TPA-BZ DG1 were higher than those of the other hyperbranched TPA-BZs, especially in terms of $P_{\mathrm{b}}(0.037 \mathrm{ppm})$. The values of $C_{\mathrm{f}}$ of $P_{\mathrm{a}}-P_{\mathrm{d}}$ of TPA-BZ tetramer were all the same $(0.007 \mathrm{ppm})$. Interestingly, the lowest values of $C_{\mathrm{f}}$ of $P_{\mathrm{a}}-P_{\mathrm{d}}$ for TPA-BZ monomer and TPA-BZ DG1 all remained constant at $0.029 \mathrm{ppm}$.

According to the concept of $H_{\mathrm{BZ}}$ and $L_{\mathrm{BZ}}$ portion analysis, the structural compositions could be selected to analyze the relationship between the $H_{\mathrm{BZ}}$ portion and the chemical constructions (conformations), using ${ }^{1} \mathrm{H}$ NMR spectra. For the ${ }^{13} \mathrm{C} N M R$ spectra, we selected a structural composition possessing a b-BZ set and its two end-connected components (e.g., nitrogen atoms and their connected b-BZ sets) to analyze the characteristic signals of $P_{\mathrm{a}}-P_{\mathrm{c}}$, and selected a structural composition of $t$-BZs containing three b-BZ sets (their one end connected to the same center nitrogen atom) to analyze the characteristic signal of $P_{\mathrm{d}}$. Table S4† summarizes the structural compositions of the hyperbranched TPA-BZs and TPA-BZ DG1 analyzed in terms of peaks $P_{\mathrm{a}}-P_{\mathrm{d}}$.

For hyperbranched TPA-BZs and TPA-BZ DG1, 14 among the 25 types of structural compositions possibly had values of $C_{\mathrm{f}}$ $\left(P_{\mathrm{a}}-P_{\mathrm{d}}\right)$ greater than zero $\left(C_{\mathrm{fs}}>0\right)$ in their ${ }^{13} \mathrm{C}$ NMR spectra, based on the analytical results in Table $\mathrm{S} 4$ (code rules and analysis progresses are provided in the ESI $\dagger$ ). Scheme 3 presents the possible mechanism behind this phenomenon for hyperbranched TPA-BZs and TPA-BZ DG1. The carbon atoms in the chemical structures of the TPA-BZ monomers labeled with a letter $x(x=\mathrm{a}, \mathrm{b}, \mathrm{c}$, or $\mathrm{d})$ are those that possibly affected the positive value of $C_{\mathrm{f}}$ for peak $x\left(\mathrm{P}_{x}\right)$. The $C_{\mathrm{fs}}>0\left(P_{\mathrm{a}}-P_{\mathrm{d}}\right)$ of TPA-BZ monomer were possibly affected by the structural compositions of [T13] $\left(P_{\mathrm{a}}, 0.058 \mathrm{ppm}\right),[\mathrm{F} 33]\left(P_{\mathrm{b}}, 0.029 \mathrm{ppm}\right),[\mathrm{F} 33]\left(P_{\mathrm{c}}, 0.029\right.$ $\mathrm{ppm})$, and [U133] $\left(P_{\mathrm{d}}, 0.029 \mathrm{ppm}\right)$. TPA-BZ trimer has no structural components that affected its $C_{\mathrm{f}}>0$, due to its linear TPA-BZ monomers. The $C_{\mathrm{fs}}>0$ of TPA-BZ tetramer were possibly affected by the structural compositions of [T12] $\left(P_{\mathrm{a}}\right.$, $0.007 \mathrm{ppm}),[\mathrm{C} 2213]\left(P_{\mathrm{b}}, 0.007 \mathrm{ppm}\right),[\mathrm{C} 3322]\left(P_{\mathrm{b}}, 0.007 \mathrm{ppm}\right)$, [F23] $\left(P_{\mathrm{c}}, 0.007 \mathrm{ppm}\right),[\mathrm{U} 123]\left(P_{\mathrm{d}}, 0.007 \mathrm{ppm}\right)$, and [U222] $\left(P_{\mathrm{d}}\right.$, $0.007 \mathrm{ppm})$. The $C_{\mathrm{fs}}>0\left(P_{\mathrm{a}}-P_{\mathrm{d}}\right)$ of TPA-BZ DG1 were possibly affected by the structural compositions of [T23] $\left(P_{\mathrm{a}}, 0.029 \mathrm{ppm}\right)$, [C332ㄹ $\left(P_{\mathrm{b}}, 0.037 \mathrm{ppm}\right),[\mathrm{UD}]\left(P_{\mathrm{d}}, 0.029 \mathrm{ppm}\right)$, and [U233] $\left(P_{\mathrm{d}}\right.$, $0.029 \mathrm{ppm})$.

Fig. 5 presents the values of $C_{\mathrm{f}}$ of $\mathrm{P}_{\mathrm{e}}-\mathrm{P}_{\mathrm{h}}$ for hyperbranched TPA-BZs and TPA-BZ DG1. The values of $C_{\mathrm{f}}$ of TPA-BZ monomer were relatively high, all greater than $0.029 \mathrm{ppm}$. The values of $C_{\mathrm{f}}$ for $P_{\mathrm{e}}$ and $P_{\mathrm{g}}$ of TPA-BZ DG1 were also relatively high, all greater than $0.036 \mathrm{ppm}$; the spectrum lacked the characteristic signal splits of $P_{\mathrm{f}}$ and $P_{\mathrm{h}}$ because of the symmetrical chemical structure of this dendrimer. For the values of $C_{\mathrm{f}}$ of TPA-BZ tetramer, those of both $P_{\mathrm{f}}$ and $P_{\mathrm{g}}$ were equal to $0.007 \mathrm{ppm}$ and those of both $P_{\mathrm{e}}$ and $P_{\mathrm{h}}$ were equal to zero. The values of $C_{\mathrm{f}}$ of TPA-BZ trimer were all zero because of the linear asymmetrical TPA-BZs. Hence, the values of $C_{\mathrm{f}}$ of both $P_{\mathrm{f}}$ and $P_{\mathrm{g}}$ in hyperbranched TPA-BZs (containing four TPA groups) would increase upon increasing the DB. The values of $C_{\mathrm{f}}$ of both $P_{\mathrm{e}}$ and $P_{\mathrm{h}}$ in hyperbranched TPA-BZs (containing four TPA groups) would remain equal to zero. In addition, the value of $C_{\mathrm{f}}$ of $P_{\mathrm{g}}$ would appear to be a good candidate for analyzing the DBs of TPA-BZ monomers from 0 to $100 \%$.

Scheme 3 presents the possible mechanism behind the trends in the signals $\mathrm{P}_{\mathrm{e}}-\mathrm{P}_{\mathrm{h}}$ for hyperbranched TPA-BZs and TPA-BZ DG1. The characteristic signals of $\mathrm{P}_{\mathrm{e}}-\mathrm{P}_{\mathrm{h}}$ in the ${ }^{13} \mathrm{C}$ NMR spectra belong to the oxazine rings of the TPA-BZ monomers, while $P_{\mathrm{f}}$ and $P_{\mathrm{h}}$ represent the characteristic signal splits arising from asymmetrical chemical structures; this behavior is similar to that of the $H_{\mathrm{Bz}}$ portion in ${ }^{1} \mathrm{H}$ NMR spectra. As a result, the signal splits of $P_{\mathrm{f}}$ and $P_{\mathrm{h}}$ of the oxazine rings can be assigned (Scheme 3) to the $\mathrm{BZ}$ rings in red (belonging to the $H_{\mathrm{BZ}}$ portion of ${ }^{1} \mathrm{H}$ NMR spectra) and the signals of $P_{\mathrm{e}}$ and $P_{\mathrm{g}}$ of the oxazine rings to the $\mathrm{BZ}$ rings in blue (belonging to the $L_{\mathrm{BZ}}$ portion of ${ }^{1} \mathrm{H}$ NMR spectra). The design strategies of the TPA-BZ chemical constructions (Scheme 2) also can be used to examine the relationship between the values of $C_{\mathrm{f}}\left(P_{\mathrm{a}}-P_{\mathrm{h}}\right)$ and the chemical construction developments of TPA-BZ monomer and TPA-BZ trimer. Scheme 3 and Table 2 present the relationship between the values of $C_{\mathrm{f}}$ and their chemical construction developments as the number of TPA and BZ groups increased for TPA-BZ monomer and TPA-BZ trimer. The values of $C_{\mathrm{f}}$ of TPA-BZ DG1 ranged from 0.029 to $0.037 \mathrm{ppm}$, very close to those of TPA-BZ monomer ( $>0.029 \mathrm{ppm})$; it is possible that TPA-BZ DG1 was composed of three monomers developed from their focal group directions, resulting in their relative higher values of $C_{\mathrm{f}}$. The 


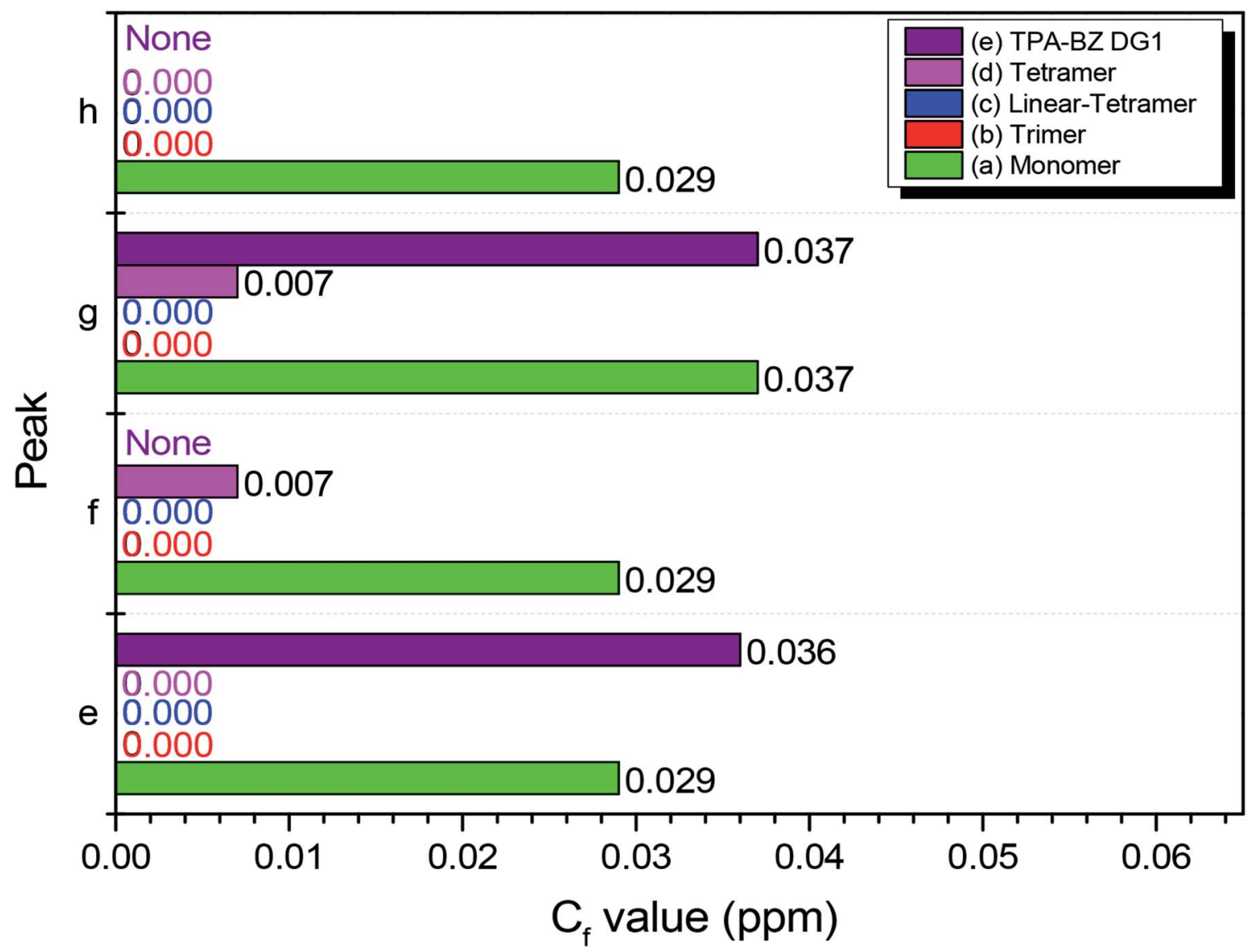

Fig. 5 Values of $C_{f}$ (peaks e-h) in ${ }^{13}$ C NMR spectra of hyperbranched TPA-BZs and TPA-BZ DG1: (a) monomer, (b) trimer, (c) linear-tetramer, (d) tetramer, and (e) TPA-BZ DG1.

linear TPA-BZ trimer was developed from TPA-BZ monomer through its terminal group directions and had values of $C_{\mathrm{f}}$ that remained at zero. The branching TPA-BZ tetramer was developed from TPA-BZ trimer through its focal group direction and had values of $C_{\mathrm{f}}$ ranging from 0 to $0.007 \mathrm{ppm}$, slightly greater than those of TPA-BZ trimer $\left(C_{\mathrm{fs}}=0\right)$. linear TPA-BZ tetramer was developed from TPA-BZ trimer through its terminal group directions; it had constant values of $C_{\mathrm{f}}$ of zero because of its linear TPA-BZ monomers. Hence, the developed directions of TPA-BZ monomer and TPA-BZ trimer strongly affected the values of $C_{\mathrm{f}}$ of the resulting compounds. Interestingly, the values of $C_{\mathrm{f}}$ could increase when developing through the direction of the focal group (e.g., TPA-BZ DG1 and TPA-BZ tetramer) or could decrease when developing through the direction of the terminal groups (e.g., trimer and lineartetramer) of TPA-BZ monomer and TPA-BZ trimer. For the TPA-BZ monomers containing four TPA groups, the values of $C_{\mathrm{f}}$ increased upon increasing the DB (Table 2); for example, they were zero for linear TPA-BZ tetramer $(\mathrm{DB}=0 \%)$, from 0 to $0.007 \mathrm{ppm}$ for TPA-BZ tetramer $(\mathrm{DB}=75 \%)$, and from 0.029 to $0.037 \mathrm{ppm}$ for TPA-BZ DG1 (DB $=100 \%)$. Hence, the values of $C_{\mathrm{f}}$ could also be used to determine the chemical constructions and conformations of the TPA-BZs. Interestingly, the linear asymmetrical TPA-BZs (trimer and linear-tetramer) could not be distinguished in terms of the values of $C_{\mathrm{f}}$ in their ${ }^{13} \mathrm{C}$ NMR spectra; instead, these linear monomers could be identified from their ${ }^{1} \mathrm{H}$ NMR spectra (through $H_{\mathrm{BZ}}$ analysis or the chemical shifts of the signals of the oxazine ring).

\section{DSC and FTIR spectroscopic analyses of hyperbranched TPA-} BZs and TPA-BZ DG1

We employed DSC to study the thermal behavior of the hyperbranched TPA-BZs and TPA-BZ DG1. Fig. 6A presents the DSC traces (first run) of the uncured hyperbranched TPA-BZs and TPA-BZ DG1. The exothermic peaks of TPA-BZ monomer, TPABZ trimer, linear TPA-BZ tetramer, TPA-BZ tetramer, and TPABZ DG1 appeared at temperatures of 236.5, 236.3, 241.0, 238.7, and $231.3{ }^{\circ} \mathrm{C}$, respectively-that is, they increased upon increasing the number of TPA units in the hyperbranched TPABZs. Interestingly, the temperatures of the exothermic peaks for the TPA-BZ containing four TPA groups decreased upon increasing the DB. For example, the temperature of exothermic peaks followed the trend TPA-BZ DG1 $(\mathrm{DB}=100 \%)<$ TPA-BZ tetramer $(\mathrm{DB}=75 \%)<$ linear TPA-BZ tetramer $(\mathrm{DB}=0 \%)$, indicating that a symmetrical chemical construction led to the exothermic peak appearing at a lower temperature. All four hyperbranched TPA-BZs provided a sharp and symmetrical exothermic peak centered in the range $236-241^{\circ} \mathrm{C}$-lower than 

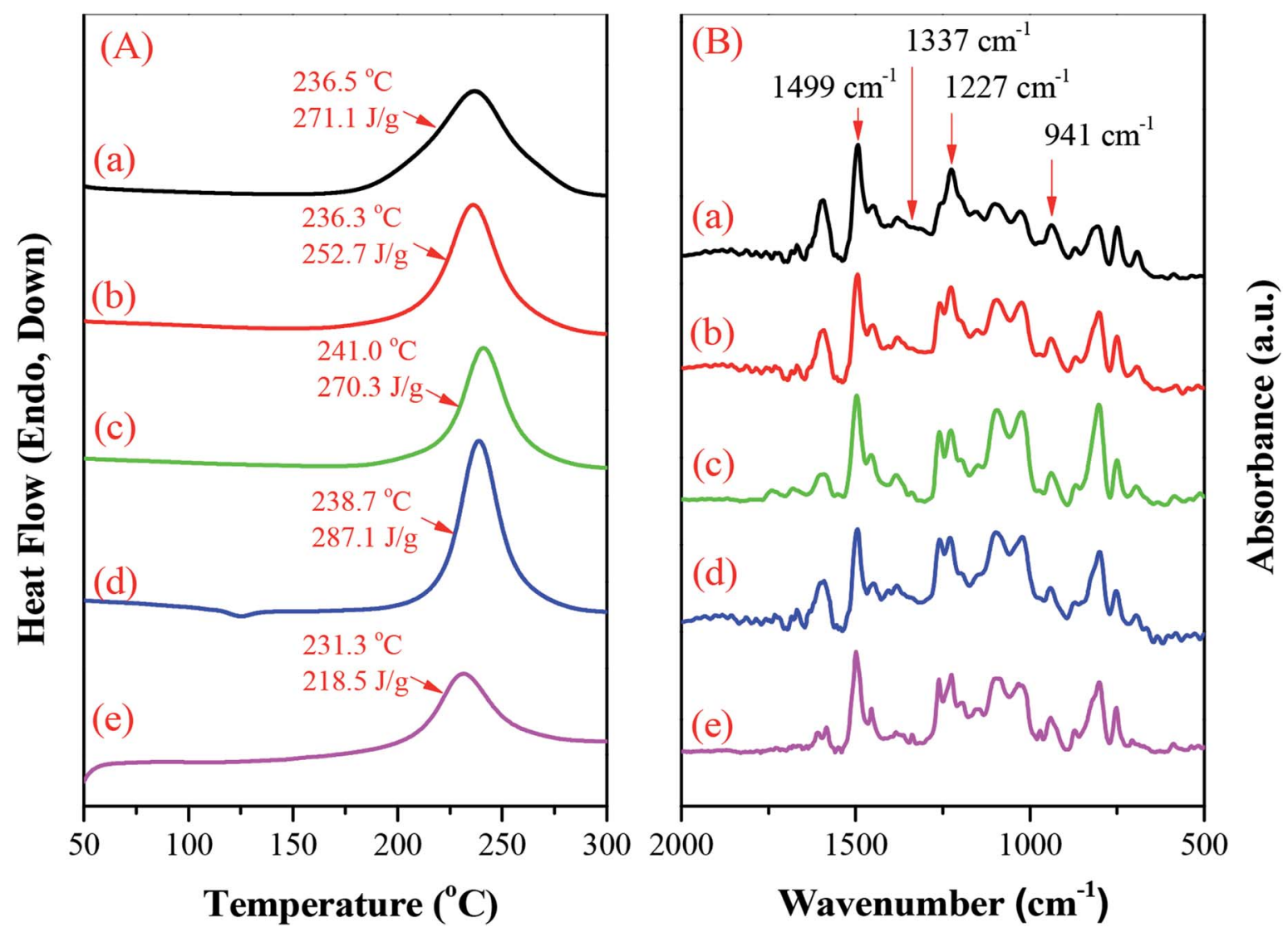

Fig. 6 (A) DSC traces and (B) FTIR spectra (recorded at room temperature) of uncured hyperbranched TPA-BZs and TPA-BZ DG1: (a) monomer, (b) trimer, (c) linear-tetramer, (d) tetramer, and (e) TPA-BZ DG1.

that of the conventional Pa-type BZ monomer (3-phenyl-3,4dihydro- $2 \mathrm{H}$-benzoxazine, $\left.263{ }^{\circ} \mathrm{C}\right)^{54}$ and the TPA derivatives featuring mono- or di-functional BZ moieties $\left(248-270{ }^{\circ} \mathrm{C}\right),{ }^{55-57}$ implying that these hyperbranched TPA-BZs all have thermal ring-opening polymerization ability and high purity, and significantly improve upon the high temperatures commonly found for ring-opening polymerization of $\mathrm{BZ}$ monomers. Interestingly, the temperatures of the exothermic peaks for the hyperbranched TPA-BZs remained higher than those of TPA-BZ dendrimers $\left(231-235{ }^{\circ} \mathrm{C}\right) .{ }^{41}$ The temperature of the exothermic peak of the linear symmetrical BZ monomer was, however, higher than that of its asymmetrical counterpart, presumably because a bulky intermediate introduced into the backbone of a symmetrical BZ monomer can move the exothermic peak to higher temperature $;^{45}$ this behavior very different from that of TPA-BZ monomers, possibly due to the branching chemical constructions of TPA-BZs and the incorporation of TPA groups into the monomers.

Fig. 6B presents FTIR spectra of the hyperbranched TPA-BZs and TPA-BZ DG1, recorded at room temperature. The characteristic adsorption peaks of the BZ moieties for these four types of hyperbranched TPA-BZs were located at 1227 (asymmetric COC stretching), 1337 ( $\mathrm{CH}_{2}$ wagging), 941 and 1499 (vibrations of trisubstituted benzene ring) $\mathrm{cm}^{-1}$, confirming the successful incorporation of the BZ groups into hyperbranched TPA-BZs.
The characteristic adsorption peaks of the BZ moieties for TPABZ DG1 were also located near 1225, 1338, 941 and $1499 \mathrm{~cm}^{-1}$. Fig. $\mathrm{S} 5 \dagger$ provides an enlarged view $\left(850\right.$ to $1000 \mathrm{~cm}^{-1}$ ) of the FTIR spectra of Fig. 6B. The absorption peak at $941 \mathrm{~cm}^{-1}$ has a shoulder peak at $928 \mathrm{~cm}^{-1}$, resulting from the asymmetrical chemical construction of the hyperbranched TPA-BZs. ${ }^{44}$ Interestingly, the spectrum of the symmetrical TPA-BZ DG1 also has a shoulder peak at $928 \mathrm{~cm}^{-1}$, similar to that of its asymmetrical counterparts-possibly resulting from peak splitting being affected only by the small region of the chemical structure arising from the asymmetrical dendrons of TPA-BZ DG1.

\section{UV-Vis absorption and PL emission spectra of hyperbranched} TPA-BZs and TPA-BZ DG1

We recorded UV-Vis absorption and PL emission spectra to investigate the photophysical properties of the hyperbranched TPA-BZs and TPA-BZ DG1 (Table S5 $†$ ). Fig. 7A presents the UVVis absorption spectra for pure TPA, hyperbranched TPA-BZs and TPA-BZ DG1 in THF at a concentration of $10^{-4} \mathrm{M}$. Fig. 7A(a) reveals the characteristic absorption peak centered at $299 \mathrm{~nm}$ for pristine TPA, ascribed to the locally excited (LE) $\pi-$ $\pi^{*}$ transition. Fig. 7A-(b) reveals three characteristic absorption peaks at 250, 277, and $304 \mathrm{~nm}$ for TPA-BZ monomer. We attribute the signal at $250 \mathrm{~nm}$ (peak 1) to the high-energy $\pi-\pi^{*}$ transition, and those at 277 and $304 \mathrm{~nm}$ (peaks 2 and 3, 


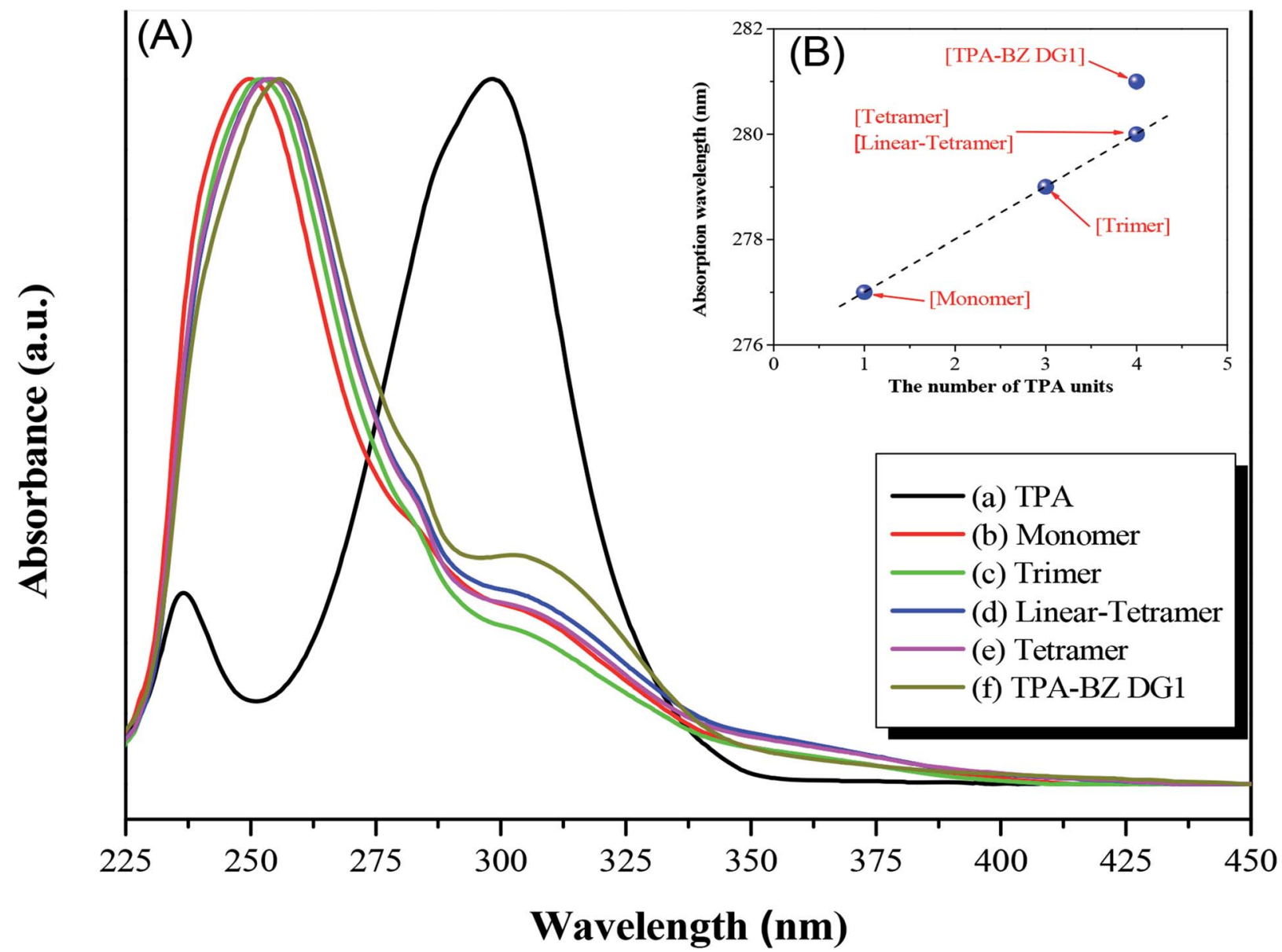

Fig. 7 (A) UV-Vis absorbance spectra and (B) absorption wavelengths plotted with respect to the number of TPA units for pristine TPA, hyperbranched TPA-BZs, and TPA-BZ DG1 as solutions in THF (concentration: $10^{-4} \mathrm{M}$ ).

respectively) to the LE $\pi-\pi^{*}$ transition and the intramolecular charge-transfer (ICT) $\pi-\pi^{*}$ transition, respectively, from the relatively strongly electron donating TPA moieties to the relatively weakly electron donating BZ moieties, as a hybridized local and charge-transfer (HLCT) transition. ${ }^{58,59}$ Upon increasing the number of TPA groups in the hyperbranched TPA-BZs, a small bathochromic shift occurred in the absorption spectrum (peak 2) in Fig. 7A, possibly the result of the increased conjugation length. ${ }^{60}$ Interestingly, the bathochromic shifts of peak 2 for linear TPA-BZ tetramer and TPA-BZ tetramer [Fig. 7A-(d) and (e)] were similar (signals at $280 \mathrm{~nm}$ ), indicating that the effect of the number of TPA groups was stronger than that of the conformations (DBs of linear TPA-BZ tetramer and TPA-BZ tetramer were 0 and $75 \%$, respectively). The bathochromic shift of peak 2 for TPA-BZ DG1 [Fig. 7A-(f)] was greater than those of linear TPA-BZ tetramer and TPA-BZ tetramer; these three $\mathrm{BZ}$ monomers have the same chemical formula $\left(\mathrm{C}_{126} \mathrm{H}_{105} \mathrm{~N}_{13} \mathrm{O}_{9}\right)$, but different DBs, especially in the symmetrical TPA-BZ dendrimers, resulting in a significant increase in the conjugation length. Prasad et al. reported that as the branching number of the TPA derivatives increased, bathochromic shifts were observed in UV-Vis absorption and onephoton emission spectra, resulting in an enhancement in the
TPA cross section, ${ }^{42}$ due to an enhancement in vibronic coupling, on the basis of a theoretical study. ${ }^{61}$ In addition, we also observed a bathochromic shift for peak 1 that was similar to that of peak 2. Fig. 7B presents the absorption wavelength in the UV-Vis spectra of the hyperbranched TPA-BZs and TPA-BZ DG1 plotted with respect to the number of TPA groups. The absorption wavelengths of these hyperbranched TPA-BZs were linearly related to the number of TPA groups, even for the different conformations of the monomers of linear TPA-BZ tetramer and TPA-BZ tetramer. TPA-BZ DG1 exhibited an absorption wavelength relatively higher than those of linear TPA-BZ tetramer and TPA-BZ tetramer, due to the significantly greater conjugation length of the TPA-BZ dendrimer $(\mathrm{DB}=$ $100 \%) .{ }^{42}$

Fig. 8A presents the PL emission spectra for pure TPA, hyperbranched TPA-BZs, and TPA-BZ DG1 in THF at a concentration of $10^{-4} \mathrm{M}$ after excitation at a wavelength of $300 \mathrm{~nm}$. Fig. 8A-(a) reveals that the emission peak was centered at $359 \mathrm{~nm}$ for pristine TPA, the result of the LE $\pi-\pi^{*}$ transition.

Fig. 8A-(b) reveals two PL emission peaks for TPA-BZ monomer at 359 and $422 \mathrm{~nm}$ (peaks L and $\mathrm{H}$, respectively), possibly the results of an HLCT transition and an aggregation occurred, respectively. Upon increasing the number of TPA 


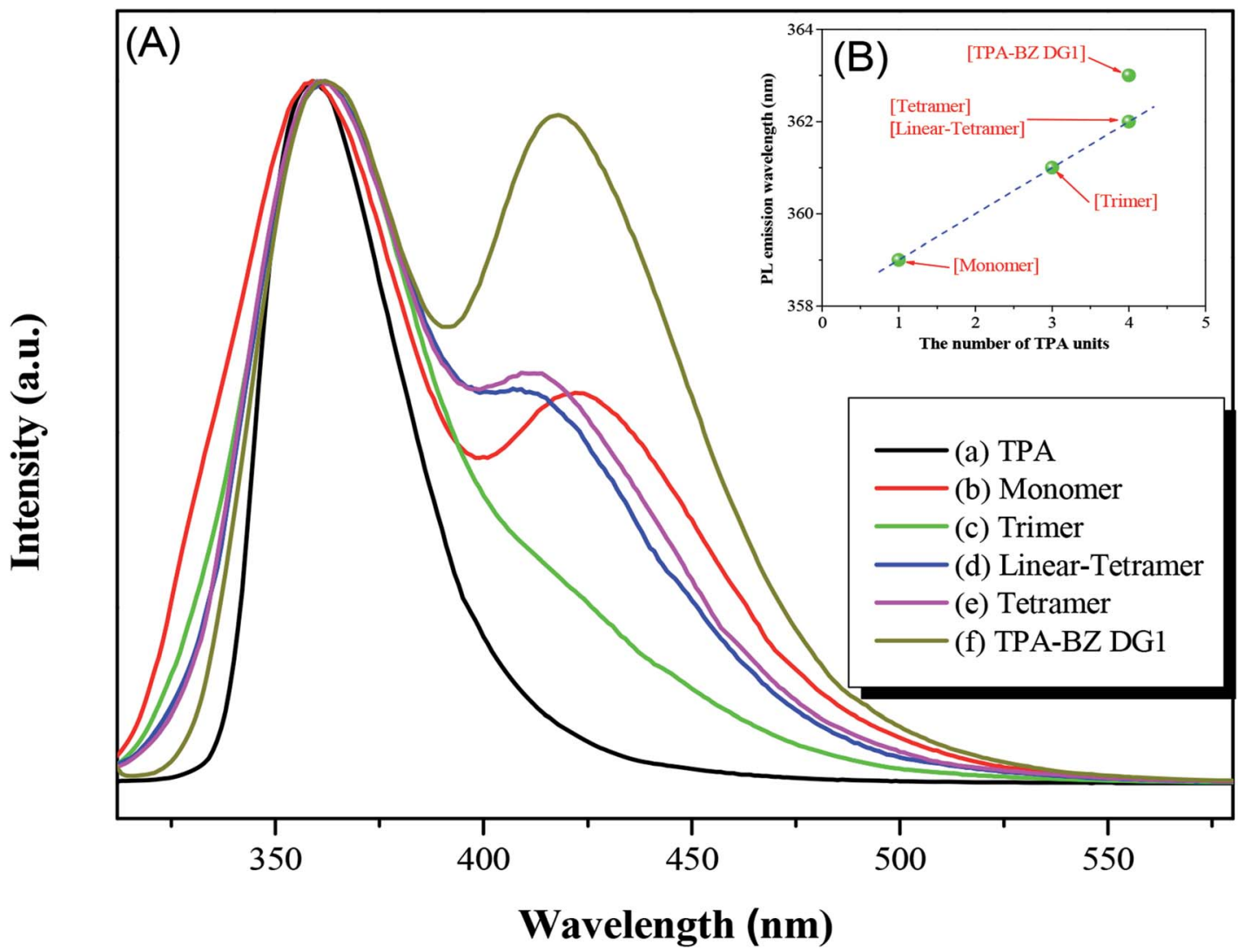

Fig. 8 (A) PL (excitation wavelength: $300 \mathrm{~nm}$ ) spectra and (B) PL emission wavelengths plotted with respect to the number of TPA units for pristine TPA, hyperbranched TPA-BZs, and TPA-BZ DG1 as solutions in THF (concentration: $10^{-4} \mathrm{M}$ ).

groups in the hyperbranched TPA-BZs, peak L in the PL emission spectrum shifted from 359 to $362 \mathrm{~nm}$ for both TPA-BZ monomer and TPA-BZ tetramer; this small bathochromic shift possibly resulted from the increase in the conjugation length. ${ }^{60}$ Interestingly, the bathochromic shifts of peak L for linear TPABZ tetramer and TPA-BZ tetramer were similar (signals at 362 $\mathrm{nm}$ ), suggesting that the effect of the number of TPA groups was stronger than that of the conformations; this phenomenon is similar to that observed in the UV-Vis spectra. In addition, peak $\mathrm{H}$ for TPA-BZ trimer had intensity lower than those of the other hyperbranched TPA-BZs, implying that TPA-BZ trimer was dispersed well in THF and less likely than the other TPA-BZ monomers to aggregate.

The bathochromic shift of peak L for TPA-BZ DG1 [Fig. 8A(f)] was greater than those of linear TPA-BZ tetramer and TPA$\mathrm{BZ}$ tetramer, similar to the behavior in the UV-Vis spectra and possibly due to the DB increasing, especially for the symmetrical TPA-BZ dendrimers, resulting in greater conjugation lengths. ${ }^{42,61}$ The intensity of peak $\mathrm{H}$ for TPA-BZ DG1 was higher than that of TPA-BZ tetramer because the symmetrical dendrimer aggregated more readily than did its asymmetrical counterpart. Fig. 8B presents the PL emission wavelengths of the hyperbranched TPA-BZs and TPA-BZ DG1 plotted with respect to the number of TPA groups. The PL emission wavelengths of these hyperbranched TPA-BZs corresponded linearly with the number of TPA groups, even for the different conformation monomers for linear TPA-BZ tetramer and TPABZ tetramer. TPA-BZ DG1 exhibited a PL emission wavelength higher than those of linear TPA-BZ tetramer and TPA-BZ tetramer, due to the greater conjugation length of the TA-BZ dendrimer. ${ }^{42}$ The UV-Vis absorption and PL emission spectra of the hyperbranched TPA-BZs featured small bathochromic shifts that were affected strongly by the number of TPA groups, presumably because of the increase in the effective conjugation length. ${ }^{41-43}$ Interestingly, the effective conjugation length of the TPA-BZs was not affected by the DB of the asymmetrical hyperbranched monomers, except in the case of the symmetrical dendrimers; for example, TPA-BZ DG1 possesses an effective conjugation length relatively higher than those of the asymmetrical hyperbranched TPA-BZs (e.g., TPA-BZ trimer and linear TPA-BZ tetramer) because the branching number increased significantly for the TPA-BZ dendrimers.

\section{Thermal ring-opening polymerization of hyperbranched TPA- BZs and TPA-BZ DG1}

The hyperbranched TPA-BZs and TPA-BZ DG1 possess the ability to undergo thermal ring-opening polymerization. Scheme $56 \dagger$ presents the possible chemical structure of the polymerized TPA-BZ tetramer. We employed DSC, FTIR spectroscopy, and TGA to study the thermal ring-opening polymerization of the hyperbranched TPA-BZs and TPA-BZ DG1. DSC 
revealed the thermal polymerization behavior of TPA-BZ monomer before and after curing at $150,180,210$, and $240{ }^{\circ} \mathrm{C}$ (Fig. 9A). Fig. 9A-a presents the DSC trace of the uncured monomer; a sharp exothermic peak was centered at $236.5{ }^{\circ} \mathrm{C}$ with a reaction heat of $271.1 \mathrm{~J} \mathrm{~g}^{-1}$. The reaction heat of the exothermic peak decreased gradually upon increasing the curing temperature, eventually disappearing at $240{ }^{\circ} \mathrm{C}$, implying that the full curing state of the polybenzoxazine had been achieved. The temperature of the exothermic peak increased upon increasing the curing temperature, presumably because the relatively higher crosslinking density of the monomer inhibited its thermal curing ability. ${ }^{35} \mathrm{Fig}$. 9A-e reveals a value of $T_{\mathrm{g}}$ of $188.4{ }^{\circ} \mathrm{C}$ after curing at $240{ }^{\circ} \mathrm{C}$, revealing an enhancement in the crosslinking density and suggesting that the full curing state of the polybenzoxazine had been achieved at $240{ }^{\circ} \mathrm{C}$. FTIR spectroscopy revealed the thermal polymerization behavior of TPA-BZ monomer before and after curing at 150, 180, 210, 220, 230, and $240{ }^{\circ} \mathrm{C}$ (Fig. 9B). The characteristic adsorption peaks of the BZ moieties for TPA-BZ monomer appeared at 1227 and $941 \mathrm{~cm}^{-1}$. The characteristic adsorption peak at $941 \mathrm{~cm}^{-1}$ (out of plane $\mathrm{C}-\mathrm{H}$ bending) disappeared completely from the spectrum of TPA-BZ monomer after curing at $240{ }^{\circ} \mathrm{C}$ (Fig. 9B-g), suggesting that the full curing stage of BZ moieties had been achieved; this behavior is consistent with the results from the
DSC analysis. Hence, we selected a curing temperature of $240{ }^{\circ} \mathrm{C}$ for our subsequent studies.

We used TGA to examine the thermal polymerization behavior of TPA-BZ monomer. Fig. 9C presents its TGA thermograms before and after curing at 150, 180, 210, and $240{ }^{\circ} \mathrm{C}$ under a $\mathrm{N}_{2}$ atmosphere; the values of $T_{\mathrm{d}}$ of TPA-BZ monomer before and after curing at these temperatures were 129.4, 253.2, $257.4,267.2$, and $299.9^{\circ} \mathrm{C}$, respectively, with char yields of 35.4, 49.3, 42.141 .9 , and $48.7 \mathrm{wt} \%$, respectively. Thus, the values of $T_{\mathrm{d}}$ and the char yields both increased upon increasing the thermal curing temperature, resulting in an increased crosslink density. Hence, TPA-BZ monomer features higher thermal stability and higher char yield comparing to the conventional Pa-type BZ monomer. ${ }^{54}$

We also employed DSC and TGA to study the thermal ringopening polymerization of the hyperbranched TPA-BZs and TPA-BZ DG1. We used DSC to study the thermal behavior of the hyperbranched TPA-BZs and TPA-BZ DG1 after curing at $240{ }^{\circ} \mathrm{C}$, recorded at a heating rate of $20{ }^{\circ} \mathrm{C} \mathrm{min}^{-1}$. Fig. $10 \mathrm{~A}$ presents the DSC traces for the hyperbranched TPA-BZs and TPA-BZ DG1 with curing at $240{ }^{\circ} \mathrm{C}$, recorded after each curing stage. The values of $T_{\mathrm{g}}$ of TPA-BZ monomer, TPA-BZ trimer, linear TPA-BZ tetramer, TPA-BZ tetramer, and TPA-BZ DG1 after curing at $240{ }^{\circ} \mathrm{C}$ were $188.4,191.3,201.1,203.5$, and
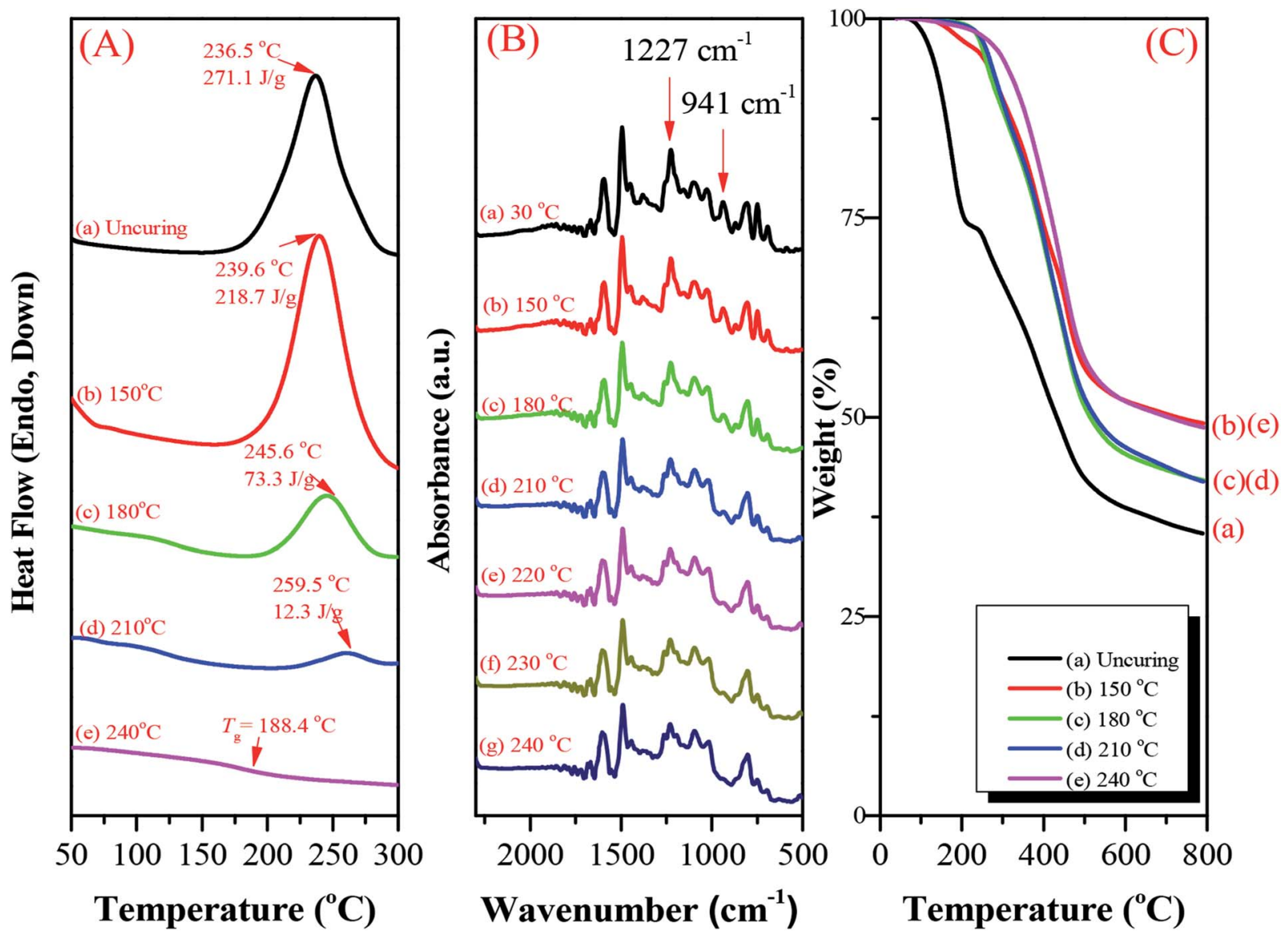

Fig. 9 (A) DSC traces, (B) FTIR spectra, and (C) TGA analysis before and after curing of TPA-BZ monomer at various temperatures, recorded after each curing stage. 

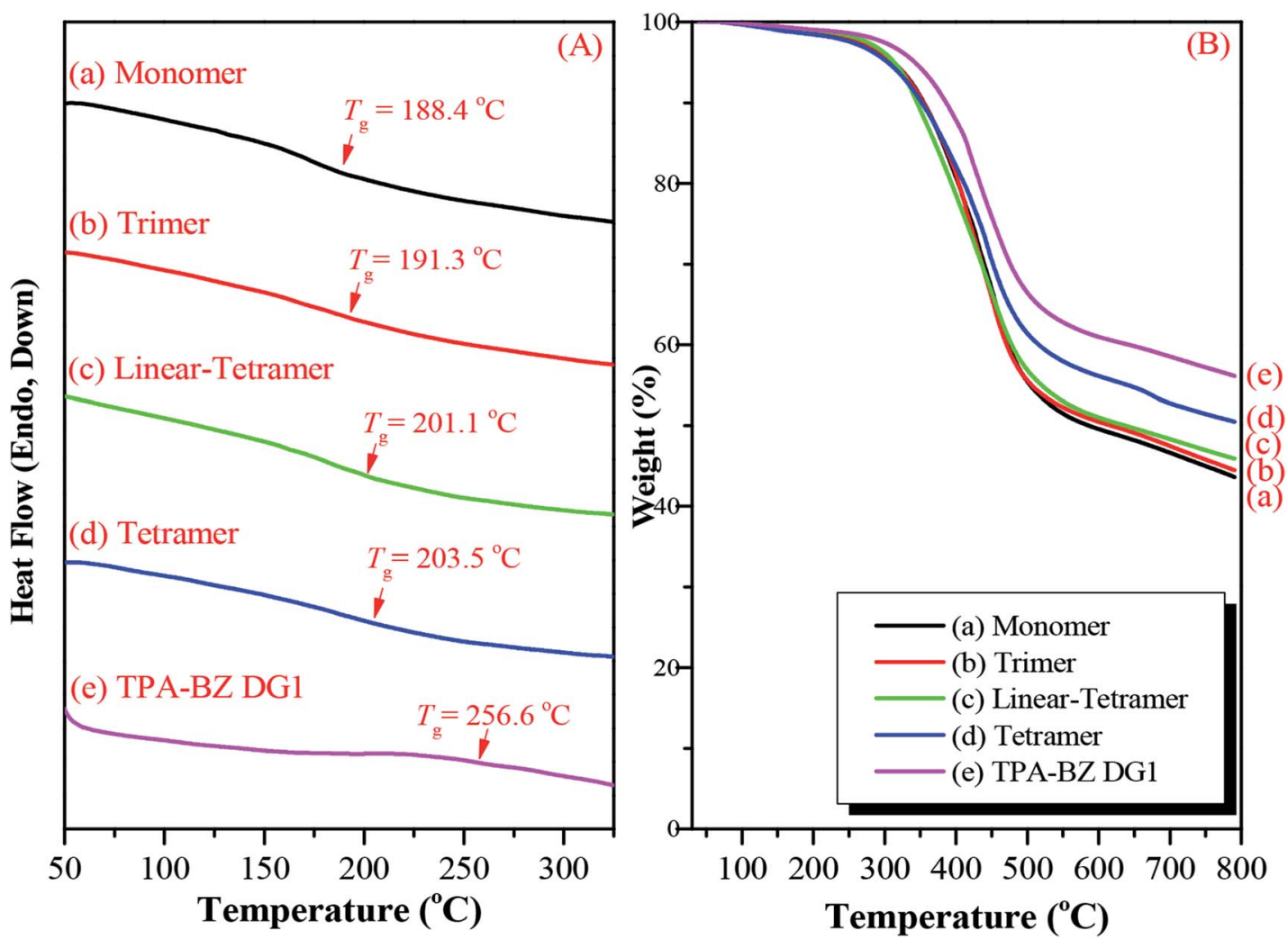

Fig. 10 (A) DSC traces and (B) TGA analysis of hyperbranched TPA-BZs and TPA-BZ DG1 after curing at $240{ }^{\circ} \mathrm{C}$ : (a) monomer, (b) trimer, (c) linear-tetramer, (d) tetramer, and (e) TPA-BZ DG1, recorded after each curing stage.

$256.6^{\circ} \mathrm{C}$, respectively. The value of $T_{\mathrm{g}}$ of TPA-BZ DG1 was higher than those of the other hyperbranched monomers because the segmental mobility in the polymer network was restricted by its dendrimer core group. ${ }^{45}$ We used TGA to investigate the thermal stability of the hyperbranched TPA-BZs and TPA-BZ DG1 after curing at $240{ }^{\circ} \mathrm{C}$ (Fig. 10B). The values of $T_{\mathrm{d}}$ and the char yields for TPA-BZ monomer, TPA-BZ trimer, linear TPA-BZ tetramer, TPA-BZ tetramer, and TPA-BZ DG1 were 311.3, 311.9, 313.0, 304.1 , and $342.0^{\circ} \mathrm{C}$, respectively, and 43.6, 44.5, 45.9, 50.5, and $56.1 \mathrm{wt} \%$, respectively. The thermal stability of TPA-BZ DG1 was greater than those of the other hyperbranched monomers, again because of its symmetrical structure.

\section{Conclusions}

We have prepared a series of well-defined thermal polymerizable hyperbranched polymers through facile one-pot Mannich condensations using a unique feeding approach. The signals representing the oxazine rings in NMR spectra revealed a tendency to shift downfield as the DB of the TPA-BZs increased, except in the case of the ${ }^{13} \mathrm{C}$ NMR spectra of the linear asymmetrical monomers. In addition, we used the structural compositions as a powerful tool to investigate the $H_{\mathrm{BZ}} / L_{\mathrm{BZ}}$ portions and values of $C_{\mathrm{f}}$ for the TPA-BZ monomers. The temperature of the exothermic peak for the thermal polymerizations of the TPA-BZ monomers decreased as the DB increased, such that it was lower than those of conventional BZ monomers; accordingly, this method overcomes the common problem of curing temperatures of $\mathrm{BZ}$ monomers being too high. UV-Vis absorption and PL emission spectra revealed bathochromic shifts of the hyperbranched TPA-BZs that increased as the number of TPA groups increased, presumably because of the greater effective conjugation length; these shifts for TPA-BZ tetramer and linear TPA-BZ tetramer were smaller than that of the symmetrical dendrimer counterpart TPA-BZ DG1, possibly because of the significant increase in effective conjugation length of the dendrimer caused by the greater branching number. DSC and FTIR spectroscopy revealed that these hyperbranched TPA-BZs underwent full curing at $240{ }^{\circ} \mathrm{C}$ to form polybenzoxazines. The polymerized TPA-BZ DG1 displayed thermal properties, in terms of values of $T_{\mathrm{g}}$ and $T_{\mathrm{d}}$, superior to those of the hyperbranched TPA-BZ polybenzoxazines because the segmental mobility in the polymer network was restricted by the dendrimer core group and because of the symmetrical construction.

\section{Conflicts of interest}

There are no conflicts to declare.

\section{Acknowledgements}

This work was financially supported by the Ministry of Science and Technology, Taiwan, under contracts MOST 106-2221-E110-067-MY3 and 105-2221-E-110-092-MY3. 


\section{References}

1 Y. H. Kim, J. Polym. Sci., Part A: Polym. Chem., 1998, 36, 16851698.

2 C. Gao and D. Yan, Prog. Polym. Sci., 2004, 29, 183-275.

3 B. I. Voit and A. Lederer, Chem. Rev., 2009, 109, 5924-5973.

4 D. A. Tomalia, A. M. Naylor and W. A. Goddard, Angew. Chem., Int. Ed., 1990, 29, 138-175.

5 C. J. Hawker and J. M. J. Fréchet, J. Am. Chem. Soc., 1990, 112, 7638-7647.

6 B. Voit, J. Polym. Sci., Part A: Polym. Chem., 2000, 38, 25052525.

7 D. Wilms, S. E. Stiriba and H. Frey, Acc. Chem. Res., 2010, 43, 129-141.

8 C. R. Yates and W. Hayes, Eur. Polym. J., 2004, 40, 1257-1281.

9 G. Maier, C. Zech, B. Voit and H. Komber, Macromol. Chem. Phys., 1998, 199, 2655-2664.

10 M. Smet, E. Schacht and W. Dehaen, Angew. Chem., Int. Ed., 2002, 41, 4547-4550.

11 W. Sinananwanich and M. Ueda, J. Polym. Sci., Part A: Polym. Chem., 2008, 46, 2689-2700.

12 W. G. Huang, L. J. Su and Z. S. Bo, J. Am. Chem. Soc., 2009, 131, 10348-10349.

13 N. Liu, J. Vignolle, J. M. Vincent, F. Robert, Y. Landais, H. Cramail and D. Taton, Macromolecules, 2014, 47, 15321542.

14 Y. Ohta, S. Fujii, A. Yokoyama, T. Furuyama, M. Uchiyama and T. Yokozawa, Angew. Chem., Int. Ed., 2009, 48, 59425945.

15 Y. Ohta, Y. Kamijyo, S. Fujii, A. Yokoyama and T. Yokozawa, Macromolecules, 2011, 44, 5112-5122.

16 D. Yang and J. Kong, Polym. Chem., 2016, 7, 5226-5232.

17 Z. B. Guan, P. M. Cotts, E. F. McCord and S. J. McLain, Science, 1999, 283, 2059-2062.

18 Z. Guan, J. Am. Chem. Soc., 2002, 124, 5616-5617.

19 C. Y. Hong, Y. Z. You, D. C. Wu, Y. Liu and C. Y. Pan, J. Am. Chem. Soc., 2007, 129, 5354-5355.

20 Y. Segawa, T. Higashihara and M. Ueda, J. Am. Chem. Soc., 2010, 132, 11000-11001.

21 M. Suzuki, A. Ii and T. Saegusa, Macromolecules, 1992, 25, 7071-7072.

22 M. Suzuki, S. Yoshida, K. Shiraga and T. Saegusa, Macromolecules, 1998, 31, 1716-1719.

23 A. Sunder, R. Hanselmann, H. Frey and R. Mulhaupt, Macromolecules, 1999, 32, 4240-4246.

24 P. Bharathi and J. S. Moore, Macromolecules, 2000, 33, 32123218.

25 A. Möck, A. Burgath, R. Hanselmann and H. Frey, Macromolecules, 2001, 34, 7692-7698.

26 R. K. Kainthan, E. B. Muliawan, S. G. Hatzikiriakos and D. E. Brooks, Macromolecules, 2006, 39, 7708-7717.

27 P. Bharathi and J. S. Moore, J. Am. Chem. Soc., 1997, 119, 3391-3392.

28 D. P. Bernal, L. Bedrossian, K. Collins and E. Fossum, Macromolecules, 2003, 36, 333-338.
29 Y. Segawa, T. Higashihara and M. Ueda, Polym. Chem., 2013, 4, 1746-1759.

30 S. Ohashi, J. Kilbane, T. Heyl and H. Ishida, Macromolecules, 2015, 48, 8412-8417.

31 W. F. Zhang, P. Froimowicz, C. R. Arza, S. Ohashi, Z. Xin and H. Ishida, Macromolecules, 2016, 49, 7129-7140.

32 N. N. Ghosh, B. Kiskan and Y. Yagci, Prog. Polym. Sci., 2007, 32, 1344-1391.

33 M. G. Mohamed, R. C. Lin, K. C. Hsu, W. Zhang, X. Zhong and S. W. Kuo, Mediated surface properties of polybenzoxazines. in Advanced and emerging polybenzoxazine science and technology, ed. $\mathrm{H}$. Ishida and $\mathrm{P}$. Froimowicz, Elsevier, Amsterdam, 2017, ch. 12, pp. 205-219.

34 M. G. Mohamed, R. C. Lin and S. W. Kuo, Polybenzoxazine/ carbon nanotube composites. in Advanced and emerging polybenzoxazine science and technology, ed. $\mathrm{H}$. Ishida and $\mathrm{P}$. Froimowicz, Elsevier, Amsterdam, 2017, ch. 36, pp. 725-738.

35 R. C. Lin, M. G. Mohamed, K. C. Hsu, J. Y. Wu, Y. R. Jheng and S. W. Kuo, RSC Adv., 2016, 6, 10683-10696.

36 R. C. Lin, M. G. Mohamed, T. Chen and S. W. Kuo, Polymers, 2017, 9, 146.

37 M. G. Mohamed and S. W. Kuo, Polymers, 2016, 8, 225.

38 H. M. Kim and B. R. Cho, Chem. Commun., 2009, 153-164.

39 Z. J. Ning and H. Tian, Chem. Commun., 2009, 5483-5495.

40 M. Pawlicki, H. A. Collins, R. G. Denning and H. L. Anderson, Angew. Chem., Int. Ed., 2009, 48, 3244-3266.

41 R. C. Lin, M. G. Mohamed and S. W. Kuo, Macromol. Rapid Commun., 2017, 1700251.

42 S. J. Chung, K. S. Kim, T. H. Lin, G. S. He, J. Swiatkiewicz and P. N. Prasad, J. Phys. Chem. B, 1999, 103, 10741-10745.

43 G. K. Paul, J. Mwaura, A. A. Argun, P. Taranekar and J. R. Reynolds, Macromolecules, 2006, 39, 7789-7792.

44 P. Yang and Y. Gu, J. Appl. Polym. Sci., 2012, 124, 2415-2422. 45 N. K. Sini and T. Endo, Macromolecules, 2016, 49, 8466-8478. 46 S. N. Kolanadiyil, M. Minami and T. Endo, Macromolecules, 2017, 50, 3476-3488.

47 C. J. Hawker, R. Lee and J. M. J. Fréchet, J. Am. Chem. Soc., 1991, 113, 4583-4588.

48 D. Hölter, A. Burgath and H. Frey, Acta Polym., 1997, 48, 3035.

49 Y. Y. Deng, Q. Zhang, H. C. Zhang, C. X. Zhang, W. H. Wang and Y. Gu, Ind. Eng. Chem. Res., 2014, 53, 1933-1939.

50 Q. Zhang, P. Yang, Y. Y. Deng, C. X. Zhang, R. Q. Zhu and Y. Gu, RSC Adv., 2015, 5, 103203-103209.

51 K. Hakala, J. M. J. Nuutinen, T. Straub, K. Rissanen and P. Vainiotalo, Rapid Commun. Mass Spectrom., 2002, 16, 1680-1685.

52 N. Nuñez-Dallos, A. Reyes and R. Quevedo, Tetrahedron Lett., 2012, 53, 530-534.

53 L. Zhang, Y. J. Zhu, D. Li, M. Wang, H. B. Chen and J. S. Wu, RSC Adv., 2015, 5, 96879-96887.

$54 \mathrm{H}$. Ishida, Overview and historical background of polybenzoxazine research. in Handbook of benzoxazine resins, ed. H. Ishida and T. Agag, Elsevier, Amsterdam, 2011, ch. 1, pp. 3-81.

55 L. C. Lin, H. J. Yen, Y. R. Kung, C. M. Leu, T. M. Lee and G. S. Liou, J. Mater. Chem. C, 2014, 2, 7796-7803. 
56 L. C. Lin, H. J. Yen, C. J. Chen, C. L. Tsai and G. S. Liou, Chem. Commun., 2014, 50, 13917-13920.

57 H. K. Shih, Y. L. Chu, F. C. Chang, C. Y. Zhu and S. W. Kuo, Polym. Chem., 2015, 6, 6227-6237.

58 J. H. Wu, W. C. Chen and G. S. Liou, Polym. Chem., 2016, 7, 61 P. Macak, Y. Luo, P. Norman and H. Ågren, J. Chem. Phys., 1569-1576.
59 C. H. Lin, Y. S. Shih, M. W. Wang, C. Y. Tseng, T. Y. Juang and C. F. Wang, RSC Adv., 2014, 4, 8692-8698.

60 Z. Q. Yan, B. Xu, Y. J. Dong, W. J. Tian and A. W. Li, Dyes Pigm., 2011, 90, 269-274.

2000, 113, 7055-7061. 